\title{
Article
}

\section{PIP-Space Valued Reproducing Pairs of Measurable Functions}

\author{
Jean-Pierre Antoine ${ }^{1, *,+}$ and Camillo Trapani ${ }^{2,+} \mathbb{D}$ \\ 1 Institut de Recherche en Mathématique et Physique, Université catholique de Louvain, \\ B-1348 Louvain-la-Neuve, Belgium \\ 2 Dipartimento di Matematica e Informatica, Università di Palermo, I-90123 Palermo, Italy; \\ camillo.trapani@unipa.it \\ * Correspondence: jean-pierre.antoine@uclouvain.be \\ + These authors contributed equally to this work.
}

Received: 16 January 2019; Accepted: 18 April 2019; Published: 30 April 2019

check for updates

\begin{abstract}
We analyze the notion of reproducing pairs of weakly measurable functions, a generalization of continuous frames. The aim is to represent elements of an abstract space $Y$ as superpositions of weakly measurable functions belonging to a space $Z:=Z(X, \mu)$, where $(X, \mu)$ is a measure space. Three cases are envisaged, with increasing generality: (i) $Y$ and $Z$ are both Hilbert spaces; (ii) $Y$ is a Hilbert space, but $Z$ is a PIP-space; (iii) $Y$ and $Z$ are both PIP-spaces. It is shown, in particular, that the requirement that a pair of measurable functions be reproducing strongly constrains the structure of the initial space $Y$. Examples are presented for each case.
\end{abstract}

Keywords: reproducing pairs; continuous frames; upper and lower semi-frames; partial inner product spaces

MSC: 41A99; 46Bxx; 46C50; 46Exx

\section{Introduction}

Representing functions in terms of simple ones, with a small number of them, if possible, is a standard problem in analysis, in particular in signal and image processing, where transmission imposes severe constraints. Signals are usually taken as square integrable functions on some manifold, hence they constitute a Hilbert space.

More precisely, given a separable Hilbert space $\mathcal{H}$, one wishes to represent an arbitrary element $f \in \mathcal{H}$ by a superposition of simpler, basic elements $\Psi=\left(\psi_{k}\right), k \in \Gamma$, with $\Gamma$ a countable index set:

$$
f=\sum_{k \in \Gamma} c_{k} \psi_{k}
$$

One usually requires that the sum converges adequately (e.g., in norm and unconditionally) and that the coefficients $c_{k}$ are unique (if possible) and easy to compute. There are many possibilities for obtaining that result, the simplest ones being that $\Psi$ be an orthonormal basis or a Riesz basis.

These two notions indeed solve the problem, but they are very rigid and lead usually to slowly converging infinite expansions. Thus frames were introduced for ensuring a better flexibility, originally in 1952 by Duffin and Schaeffer [1] in the context of nonharmonic analysis. The notion was revived by Daubechies, Grossmann and Meyer [2] in the context of wavelet theory and then became a very popular topic, in particular in Gabor and wavelet analysis [3-6]. The reason is that a good frame in a Hilbert space (that is, a frame where the ratio of the frame bounds is close to 1) is almost as good as an orthonormal basis for expanding arbitrary elements (albeit non-uniquely) and is often easier to 
construct and has in many cases additional structural properties. Let us first recall that a sequence $\Psi=\left(\psi_{k}\right)$ is a discrete frame for a Hilbert space $\mathcal{H}$ if there exist constants $0<\mathrm{m} \leq \mathrm{M}<\infty$ (the frame bounds) such that

$$
\mathrm{m}\|f\|^{2} \leq \sum_{k \in \Gamma}\left|\left\langle f \mid \psi_{k}\right\rangle\right|^{2} \leq \mathrm{M}\|f\|^{2}, \forall f \in \mathcal{H}
$$

The frame bounds are usually denoted by $A$ and $B$ in the literature, but this conflicts with the notation for operators. Here we follow our monograph [7].

As a matter of fact, most frames considered in applications are discrete, for instance in signal or image processing [4]. Yet continuous frames offer interesting mathematical problems. They have been introduced originally by Ali, Gazeau and one of us [8,9] and also, independently, by Kaiser [10]. Since then, many papers dealt with various aspects of the concept, see for instance [11-14].

However, there are situations where it is impossible to satisfy both frame bounds at the same time. Indeed, the two bounds are independent: A sequence that verifies the upper bound may not have a lower bound and a sequence that verifies the lower bound may be unbounded. To give a (simple) example, take an orthonormal basis $\left(e_{k}, k \in \mathbb{N}\right)$. Then the sequence $\left(\frac{1}{k} e_{k}\right)$ satisfies the upper bound, but has no lower bound, whereas the sequence $\left(k e_{k}\right)$ satisfies the lower bound, but not the upper one. Therefore, several generalizations of frames have been introduced. Semi-frames $[15,16]$, for example, are obtained when functions only satisfy one of the two frame bounds. Thus one speaks of upper or lower semi-frames. It turns out that a large portion of frame theory can be extended to this larger framework.

More recently, a new generalization of frames was introduced by Balazs and Speckbacher [17], called reproducing pairs. Here, given a measure space $(X, \mu)$, one considers a pair of weakly measurable functions $(\psi, \phi)$, instead of a single mapping, and one studies the correlation between the two (a precise definition is given below). This definition also includes the original definition of a continuous frame $[8,9]$ to which it reduces when $\psi=\phi$. The choice of the mappings $\psi$ and $\phi$ gives more freedom, but it leads to the problem of characterizing the range of the analysis operators, which in general may not be contained in $L^{2}(X, \mathrm{~d} \mu)$, as in the frame case. Therefore, it is natural to extend the theory to the case where the weakly measurable functions take their values in a partial inner product space (PIP-space) [7]. Actually we will go further and consider a more general construction. Namely we want to represent elements of an abstract space $f \in Y$ by weakly measurable functions $\left(C_{\psi} f\right)(x):=\left\langle f \mid \psi_{x}\right\rangle$, belonging to a space $Z(X, \mu)$, in such a way that the (formal) inner product

$$
\left\langle C_{\psi} f \mid C_{\phi} g\right\rangle=\int_{X}\left(C_{\psi} f\right)(x) \overline{\left(C_{\phi} g\right)(x)} \mathrm{d} \mu(x)
$$

is well defined. The interesting point is that the construction extends naturally from the simple case of a frame to the more general case just outlined, and this is the rationale behind the present paper. Nevertheless, since the techniques needed in this general case are very similar to those used in the previous ones, we have sketched them in these simpler cases first, in order to make the paper self-contained. Further information may be found in the original papers or in the review paper [18] that we follow closely.

The paper is organized as follows. In Section 2, we review the notions of frames, semi-frames and reproducing pairs and we recall their salient properties. In Section 3, we consider the case where $Y$ and $Z$ are both Hilbert spaces, which corresponds to genuine frames. In particular we construct and analyze the so-called coefficient spaces $W_{\psi}(X, \mu), W_{\phi}(X, \mu)$, which are Hilbert spaces in conjugate duality when $(\psi, \phi)$ is a reproducing pair. In Section 4 , motivated by the relation (3), we take for target space a PIP-space $V_{J} \sim\left\{V_{p}(X, \mu), p \in J\right\}$, in particular a lattice of Hilbert or Banach spaces (LHS/LBS). In Section 5, we go one step further and take for the initial space another PIP-space $Y_{U} \sim\left\{Y_{u}, u \in U\right\}$. In particular, we see how the requirement of having a reproducing pair affects the structure of the initial space $Y$. Finally, we conclude by an Appendix A, in which we recall the main definitions and notations about PIP-spaces (LHS/LBS) and operators on them. 


\section{Preliminaries}

Before proceeding, we list our definitions and conventions. We work in a (separable) Hilbert space $\mathcal{H}$, with the inner product $\langle\cdot \mid \cdot\rangle$ linear in the first factor. If $A$ is an operator on $\mathcal{H}$, we denote its domain by $D(A)$, its range by $\operatorname{Ran}(A)$ and its kernel by $\operatorname{Ker}(A)$. The set of all invertible bounded operators on $\mathcal{H}$ with bounded inverse is denoted $G L(\mathcal{H})$. We will consider throughout weakly measurable functions $\psi: X \rightarrow \mathcal{H}$, i.e., $\left\langle f \mid \psi_{x}\right\rangle$ is $\mu$-measurable for every $f \in \mathcal{H}$. Here $(X, \mu)$ is a locally compact, $\sigma$-compact space with a Radon measure $\mu$.

The weakly measurable function $\psi$ is called continuous frame if there exist constants $\mathrm{m}>0$ and $\mathrm{M}<\infty$ (the frame bounds) such that

$$
\mathrm{m}\|f\|^{2} \leq \int_{X}\left|\left\langle f \mid \psi_{x}\right\rangle\right|^{2} \mathrm{~d} \mu(x) \leq \mathrm{M}\|f\|^{2}, \forall f \in \mathcal{H}
$$

Remark 1. The geometry of the Hilbert space imposes a number of constraints that severely limit the existence of continuous families acting as bases or frames. Apart from the case of a separable Hilbert space where orthonormal or Riesz bases cannot be uncountable, there are more general situations where, for instance, Riesz bases cannot be continuous, but they are necessarily discrete, in a certain sense [19-22]. Nevertheless, this result is essentially of theoretical nature and does not affect the interest for continuous frames in applications. On the other hand, continuous frames do really exist in more general frameworks (rigged Hilbert space, PIP-space) as shown in [23-25] and in the present paper. This fact constitutes a further motivation for going beyond Hilbert spaces.

Given the continuous frame $\psi$, we define the analysis operator $C_{\psi}: \mathcal{H} \rightarrow L^{2}(X, \mathrm{~d} \mu)$ as

$$
\left(C_{\psi} f\right)(x)=\left\langle f \mid \psi_{x}\right\rangle, f \in \mathcal{H},
$$

and the corresponding synthesis operator $C_{\psi}^{*}: L^{2}(X, \mathrm{~d} \mu) \rightarrow \mathcal{H}$ as

$$
C_{\psi}^{*} \xi=\int_{X} \xi(x) \psi_{x} \mathrm{~d} \mu(x), \text { for } \xi \in L^{2}(X, \mathrm{~d} \mu)
$$

the integral being understood in the weak sense. Clearly both operators are bounded. We set $S_{\psi}:=C_{\psi}^{*} C_{\psi}$, i.e.,

$$
\left\langle f \mid S_{\psi} f\right\rangle=\int_{X}\left|\left\langle f \mid \psi_{x}\right\rangle\right|^{2} \mathrm{~d} \mu(x) .
$$

Thus the so-called frame or resolution operator $S_{\psi}$ is self-adjoint, invertible, bounded with bounded inverse $S_{\psi}^{-1}$, that is, $S_{\psi} \in G L(\mathcal{H})$.

In particular, if $X$ is a discrete set with $\mu$ being a counting measure, we recover the standard definition (2) of a (discrete) frame [1,3,4].

The weakly measurable function $\psi$ is said to be $\mu$-total if $\left\langle g \mid \psi_{x}\right\rangle=0$, $\mu$-a.e., implies $g=0$, that is, $\operatorname{Ker} C_{\phi}=\{0\}$. Following [15,16], we will say that a family $\Psi=\left\{\psi_{x}\right\}$ is an upper (resp. lower) semi-frame, if it is $\mu$-total in $\mathcal{H}$ and satisfies the upper (resp. lower) frame inequality. For the sake of completeness, we recall the definitions. A weakly measurable function $\psi$ is an upper semi-frame if there exists $M<\infty$ such that

$$
0<\int_{X}\left|\left\langle f \mid \psi_{x}\right\rangle\right|^{2} \mathrm{~d} \mu(x) \leq \mathrm{M}\|f\|^{2}, \forall f \in \mathcal{H}, f \neq 0 .
$$

Note that an upper semi-frame is also called a (total) Bessel mapping in the literature [13].

On the other hand, a measurable function $\phi$ is called a lower semi-frame if it satisfies the lower frame condition, that is, there exists a constant $m>0$ such that

$$
\mathrm{m}\|f\|^{2} \leq \int_{X}\left|\left\langle f \mid \phi_{x}\right\rangle\right|^{2} \mathrm{~d} \mu(x), \forall f \in \mathcal{H}
$$

Clearly, (9) implies that the function $\phi$ is $\mu$-total in $\mathcal{H}$. 
If $\psi$ is a measurable function, the operator $C_{\psi}$, formally defined as for frames, takes values in the space of measurable functions on $(X, \mu)$ and the adjoint $C_{\psi}^{*}$ is, in general, meaningless. $C_{\psi}$ has a natural domain in Hilbert space, namely,

$$
D\left(C_{\psi}\right)=\left\{f \in \mathcal{H}: C_{\psi} f \in L^{2}(X, \mathrm{~d} \mu)\right\}
$$

but this domain, in general, is not dense and nothing guarantees that it does not reduce to $\{0\}$.

If the measurable function $\psi$ is an upper semi-frame, the definition implies that $\operatorname{Ran} C_{\psi}$ is contained in $L^{2}(X, \mathrm{~d} \mu)$. If a measurable function $\phi$ is a lower semi-frame, $C_{\phi}$ is injective and has closed range.

In the lower case, the definition of the frame operator $S_{\phi}$ must be changed, since $C_{\phi}$ need not be densely defined, so that $C_{\phi}^{*}$ may not exist. Instead, following [15] (Section 2) one defines the synthesis operator as

$$
D_{\phi} F=\int_{X} F(x) \phi_{x} \mathrm{~d} \mu(x)
$$

on the domain of all elements $F \in L^{2}(X, \mathrm{~d} \mu)$ for which the integral in (10) converges weakly in $\mathcal{H}$, and then $S_{\phi}:=D_{\phi} C_{\phi}$. With this definition, it is shown in [15] (Section 2) that $S_{\phi}$ is unbounded and $S_{\phi}^{-1}$ is bounded.

All these objects are studied in detail in our previous papers [15,16]. In particular, it is shown there that a natural notion of duality exists, namely, two weakly measurable functions $\psi, \phi$ are dual to each other (the relation is symmetric) if one has

$$
\langle f \mid g\rangle=\int_{X}\left\langle f \mid \psi_{x}\right\rangle\left\langle\phi_{x} \mid g\right\rangle \mathrm{d} \mu(x), \forall f, g \in \mathcal{H}
$$

A new generalization of frames was introduced recently by Balazs and Speckbacher [17], namely, reproducing pairs. Given a measure space $(X, \mu)$, one considers a couple of weakly measurable functions $(\psi, \phi)$, instead of a single mapping. The advantage is that no further conditions are imposed on these functions, which results in an increased flexibility.

More precisely, the couple of weakly measurable functions $(\psi, \phi)$ is called a reproducing pair $[17,26]$ if (i) the sesquilinear form

$$
\Omega_{\psi, \phi}(f, g)=\int_{X}\left\langle f \mid \psi_{x}\right\rangle\left\langle\phi_{x} \mid g\right\rangle \mathrm{d} \mu(x)
$$

is well-defined and bounded on $\mathcal{H} \times \mathcal{H}$, that is, $\left|\Omega_{\psi, \phi}(f, g)\right| \leq c\|f\|\|g\|$, for some $c>0$ and every $f, g \in \mathcal{H}$; and (ii) the corresponding bounded (resolution) operator $S_{\psi, \phi}$ belongs to $G L(\mathcal{H})$.

Under these hypotheses, one has

$$
S_{\psi, \phi} f=\int_{X}\left\langle f \mid \psi_{x}\right\rangle \phi_{x} \mathrm{~d} \mu(x), \forall f \in \mathcal{H}
$$

the integral on the r.h.s. being defined in weak sense. If $\psi=\phi$, we recover the notion of continuous frame, as introduced in $[8,9]$, so that we have indeed a genuine generalization of the latter.

Notice that, if $\psi \neq \phi$, the frame operator $S_{\psi, \phi}$ is in general neither positive, nor self-adjoint, since $S_{\psi, \phi}^{*}=S_{\phi, \psi}$. However, if $(\psi, \phi)$ is a reproducing pair, then $\left(\psi, S_{\psi, \phi}^{-1} \phi\right)$ is also a reproducing pair, for which the corresponding frame operator is the identity, that is, $\psi$ and $S_{\psi, \phi}^{-1} \phi$ are in duality. Thus, there is no restriction of generality to assume that $S_{\phi, \psi}=I$ [17]. The only thing that can happen is to replace some norms by equivalent ones.

\section{The Case $Y=\mathcal{H}$ and $Z=L^{2}(X, \mu)$, Both Hilbert Spaces}

As mentioned in Section 1, we begin with the case where the initial space $Y=\mathcal{H}$ and the target space $Z=L^{2}(X, \mu)$ are both Hilbert spaces, which is characteristic of a reproducing pair as originally defined [17]. 
Given a weakly measurable function $\phi$, let us denote by $K_{\phi}(X, \mu)$ the space of all measurable functions $\xi: X \rightarrow \mathbb{C}$ such that the integral $\int_{X} \xi(x)\left\langle\phi_{x} \mid g\right\rangle \mathrm{d} \mu(x)$ exists for every $g \in \mathcal{H}$ and defines a bounded conjugate linear functional on $\mathcal{H}$, i.e., $\exists c>0$ such that

$$
\left|\int_{X} \xi(x)\left\langle\phi_{x} \mid g\right\rangle \mathrm{d} \mu(x)\right| \leq c\|g\|, \forall g \in \mathcal{H}
$$

Clearly, if $(\psi, \phi)$ is a reproducing pair, all functions $\xi(x)=\left\langle f \mid \psi_{x}\right\rangle=\left(C_{\psi} f\right)(x)$ belong to $K_{\phi}(X, \mu)$.

By the Riesz lemma, we can define a linear map $T_{\phi}: K_{\phi}(X, \mu) \rightarrow \mathcal{H}$, which we call the synthesis operator, by the weak relation

$$
\left\langle T_{\phi} \xi \mid g\right\rangle=\int_{X} \xi(x)\left\langle\phi_{x} \mid g\right\rangle \mathrm{d} \mu(x), \forall \xi \in K_{\phi}(X, \mu), g \in \mathcal{H}
$$

Next, we define the vector space

$$
W_{\phi}(X, \mu)=K_{\phi}(X, \mu) / \operatorname{Ker} T_{\phi}
$$

and equip it with the norm

$$
\left\|\xi_{\phi}\right\|_{\phi}:=\sup _{\|g\| \leq 1}\left|\int_{X} \xi(x)\left\langle\phi_{x} \mid g\right\rangle \mathrm{d} \mu(x)\right|=\sup _{\|g\| \leq 1}\left|\left\langle T_{\phi} \xi \mid g\right\rangle\right|,
$$

where we have put $\xi_{\phi}=\xi+\operatorname{Ker} T_{\phi}$ for $\xi \in K_{\phi}(X, \mu)$. Clearly, $W_{\phi}(X, \mu)$ is a normed space, called the coefficient space of $\phi$. However, the norm $\|\cdot\|_{\phi}$ derives from an inner product. First, the map $\widehat{T}_{\phi}: W_{\phi}(X, \mu) \rightarrow \mathcal{H}, \widehat{T}_{\phi} \xi_{\phi}:=T_{\phi} \xi$ is an isometry of $W_{\phi}(X, \mu)$ into $\mathcal{H}$. Next, one defines on $W_{\phi}(X, \mu)$ an inner product by setting

$$
\left\langle\xi_{\phi} \mid \eta_{\phi}\right\rangle_{(\phi)}:=\left\langle\widehat{T}_{\phi} \xi_{\phi} \mid \widehat{T}_{\phi} \eta_{\phi}\right\rangle, \xi_{\phi}, \eta_{\phi} \in W_{\phi}(X, \mu) .
$$

Finally, the norm defined by $\langle\cdot \mid \cdot\rangle_{(\phi)}$ coincides with the norm $\|\cdot\|_{\phi}$ defined in (16), since one has indeed

$$
\left\|\xi_{\phi}\right\|_{(\phi)}=\left\|\widehat{T}_{\phi} \xi_{\phi}\right\|=\left\|T_{\phi} \xi\right\|=\sup _{\|g\| \leq 1}\left|\left\langle T_{\phi} \xi \mid g\right\rangle\right|=\left\|\xi_{\phi}\right\|_{\phi}
$$

Thus $W_{\phi}(X, \mu)$ is a pre-Hilbert space with inner product $\left\langle\xi_{\phi} \mid \eta_{\phi}\right\rangle_{\phi}=\left\langle T_{\phi} \xi \mid T_{\phi} \eta\right\rangle$.

We denote by $W_{\phi}(X, \mu)^{*}$ the Hilbert dual space of $W_{\phi}(X, \mu)$, that is, the set of continuous linear functionals on $W_{\phi}(X, \mu)$. The structure of this dual will be elucidated below. The (dual) norm $\|\cdot\|_{\phi^{*}}$ of $W_{\phi}(X, \mu)^{*}$ is defined, as usual, by

$$
\|F\|_{\phi^{*}}=\sup _{\left\|\xi_{\phi}\right\|_{\phi} \leq 1}\left|F\left(\xi_{\phi}\right)\right|
$$

Now we define a conjugate linear map $\widehat{C}_{\phi}: \mathcal{H} \rightarrow W_{\phi}(X, \mu)^{*}$ by

$$
\left(\widehat{C}_{\phi} f\right)\left(\xi_{\phi}\right):=\int_{X} \xi(x)\left\langle\phi_{x} \mid f\right\rangle \mathrm{d} \mu(x), f \in \mathcal{H} .
$$

Notice that this map $\widehat{C}_{\phi}$ is conjugate linear, so it should not be confused with the linear map $C_{\psi}$ introduced in (5).

Of course, (18) means that $\left(\widehat{C}_{\phi} f\right)\left(\xi_{\phi}\right)=\left\langle T_{\phi} \xi \mid f\right\rangle=\left\langle\widehat{T}_{\phi} \xi_{\phi} \mid f\right\rangle$, for every $f \in \mathcal{H}$. Thus $\widehat{C}_{\phi}=\widehat{T}_{\phi}^{*}$, the adjoint map of $\widehat{T}_{\phi}$. By (13) it follows that $\widehat{C}_{\phi}$ is continuous . This implies that

$$
\mathcal{H}=\left[\operatorname{Ran} \widehat{T}_{\phi}\right]^{\sim} \oplus \operatorname{Ker} \widehat{C}_{\phi},
$$

where the first summand denotes the closure of Ran $\widehat{T}_{\phi}$. Hence $\widehat{C}_{\phi}^{*}=\widehat{T}_{\phi}^{* *}=\widehat{T}_{\phi}$, if $W_{\phi}(X, \mu)$ is complete. 
By modifying in an obvious way the definition given in Section 2, we say that $\phi$ is $\mu$-total if $\operatorname{Ker} \widehat{C}_{\phi}=\{0\}$.

A first preliminary result reads as follows.

Proposition 1. If $(\psi, \phi)$ is a reproducing pair, then $\operatorname{Ran} \widehat{T}_{\phi}=\mathcal{H}$.

For the proof, notice that, since $S_{\psi, \phi} \in G L(\mathcal{H})$, for every $h \in \mathcal{H}$, there exists a unique $f \in \mathcal{H}$ such that $S_{\psi, \phi} f=h$. By (12), we can conclude that

$$
\langle h \mid g\rangle=\int_{X}\left\langle f \mid \psi_{x}\right\rangle\left\langle\phi_{x} \mid g\right\rangle \mathrm{d} \mu(x), \forall g \in \mathcal{H}
$$

that is, $h=\widehat{T}_{\phi}\left[C_{\psi} f\right]_{\phi} \in \operatorname{Ran} \widehat{T}_{\phi}$. Notice that, if $(\psi, \phi)$ is a reproducing pair, both functions are necessarily $\mu$-total, which already implies that Ran $\widehat{T}_{\phi}$ is dense in $\mathcal{H}$, by (19).

For future use, we note that, if $(\psi, \phi)$ is a reproducing pair, then we have the relation [26] (Theorem 3.12).

$$
K_{\phi}(X, \mu)=\operatorname{Ran} C_{\psi} \oplus \operatorname{Ker} T_{\phi}
$$

The crucial step for characterizing the elements of $W_{\phi}(X, \mu)^{*}$ is the following result.

Theorem 1. If $(\psi, \phi)$ is a reproducing pair, then every bounded linear functional $F$ on $W_{\phi}(X, \mu)$, i.e., $F \in$ $W_{\phi}(X, \mu)^{*}$, can be represented as

$$
F\left(\xi_{\phi}\right)=\int_{X} \xi(x) \overline{\eta(x)} \mathrm{d} \mu(x), \forall \xi_{\phi} \in W_{\phi}(X, \mu)
$$

with $\eta \in K_{\psi}(X, \mu)$. The residue class $\eta_{\psi} \in W_{\psi}(X, \mu)$ is uniquely determined.

Proof. We proceed in several steps. First, if $F$ is a continuous linear functional on $W_{\phi}(X, \mu)$, then there exists a unique $g \in \mathcal{H}$, such that

$$
F\left(\xi_{\phi}\right)=\int_{X} \xi(x)\left\langle\phi_{x} \mid g\right\rangle \mathrm{d} \mu(x), \forall \xi \in K_{\phi}(X, \mu)
$$

Indeed, given $F \in W_{\phi}(X, \mu)^{*}$, there exists $c>0$ such that

$$
\left|F\left(\xi_{\phi}\right)\right| \leq c\left\|\xi_{\phi}\right\|_{\phi}=c\left\|T_{\phi} \xi\right\|, \forall \xi \in K_{\phi}(X, \mu) .
$$

Let $\widetilde{F}$ be the continuous functional on $\mathcal{H}$ defined by

$$
\widetilde{F}\left(T_{\phi} \xi\right):=F\left(\xi_{\phi}\right), \xi \in K_{\phi}(X, \mu) .
$$

The functional $\widetilde{F}$ is well-defined. Indeed, if $T_{\phi} \xi=T_{\phi} \xi^{\prime}$, then $\xi-\xi^{\prime} \in \operatorname{Ker} T_{\phi}$. Hence, $\xi_{\phi}=\xi_{\phi}^{\prime}$ and $F\left(\xi_{\phi}\right)=F\left(\xi_{\phi}^{\prime}\right)$. Thus there exists a unique $g \in \mathcal{H}$ such that

$$
\widetilde{F}\left(T_{\phi} \xi\right)=\left\langle\widehat{T}_{\phi} \xi_{\phi} \mid g\right\rangle=\int_{X} \xi(x)\left\langle\phi_{x} \mid g\right\rangle \mathrm{d} \mu(x)
$$

and $\|g\|=\|\widetilde{F}\|$. In conclusion, (22) holds true and $\|F\|_{\phi^{*}}=\|g\|$, where $\|\cdot\|_{\phi^{*}}$ is the dual norm defined in (17).

Conversely, every $g \in \mathcal{H}$ obviously defines a continuous linear functional $F$ by (22) since $\left|F\left(\xi_{\phi}\right)\right| \leq$ $\|g\|\left\|\xi_{\phi}\right\|_{\phi}$ for every $\xi_{\phi} \in W_{\phi}(X, \mu)$.

Finally, since $\eta(x):=\left\langle g \mid \phi_{x}\right\rangle \in K_{\psi}(X, \mu)$, we have the representation (21). Uniqueness is immediate. 
Remark 2. Several of the previous statements hold true without the assumption that $(\psi, \phi)$ is a reproducing pair, see [26] (Section 3) for details.

The lesson of Theorem 1 is that the map

$$
\Lambda: F \in W_{\phi}(X, \mu)^{*} \mapsto \eta_{\psi} \in W_{\psi}(X, \mu)
$$

is well-defined and conjugate linear. On the other hand, $\Lambda(F)=\Lambda\left(F^{\prime}\right)$ implies easily $F=F^{\prime}$. Therefore $W_{\phi}(X, \mu)^{*}$ can be identified with a closed subspace of $\overline{W_{\psi}}(X, \mu):=\left\{\overline{\xi_{\psi}}: \xi \in K_{\psi}(X, \mu)\right\}$, where the overbar denotes the complex conjugate.

Actually, there is more: if $(\psi, \phi)$ is a reproducing pair, $W_{\phi}(X, \mu)^{*}$ and $\overline{W_{\psi}}(X, \mu)$ can be identified. The proof relies on two technical lemmas which can be found in [26] (Lemmas 3.10 and 3.11). These imply that the map $\Lambda$ defined in (23) is surjective. Hence, $W_{\phi}(X, \mu)^{*} \simeq \overline{W_{\psi}}(X, \mu)$, where $\simeq$ denotes a bounded isomorphism and the norm $\|\cdot\|_{\psi}$ is the dual norm of $\|\cdot\|_{\phi}$. Finally, for every $\eta \in W_{\psi}(X, \mu)$, there exists $g \in \mathcal{H}$ such that $\eta(x)=\left\langle\phi_{x} \mid g\right\rangle$. In conclusion, one obtains the main result of [26], namely:

Theorem 2. If $(\psi, \phi)$ is a reproducing pair, the spaces $W_{\phi}(X, \mu)$ and $W_{\psi}(X, \mu)$ are both Hilbert spaces, conjugate dual of each other with respect to the sesquilinear form

$$
\langle\xi \mid \eta\rangle:=\int_{X} \xi(x) \overline{\eta(x)} \mathrm{d} \mu(x),
$$

which coincides with the inner product of $L^{2}(X, \mu)$ whenever the latter makes sense.

This is true, in particular, for $\phi=\psi$, since then $\psi$ is a continuous frame and $W_{\psi}(X, \mu)$ is a closed subspace of $L^{2}(X, \mu)$.

In addition, the converse of Theorem 2 holds if $\phi$ and $\psi$ are both $\mu$-total: in that case, $(\psi, \phi)$ is a reproducing pair if and only if $W_{\phi}(X, \mu)$ and $W_{\psi}(X, \mu)$ are Hilbert spaces, conjugate dual of each other with respect to the sesquilinear form (24).

In fact, there is more. In a recent paper [22], it is shown that $W_{\phi}(X, \mu)$ (and thus also $W_{\psi}(X, \mu)$, by symmetry), is a reproducing kernel Hilbert space (RKHS) [27]. In order to see this, take an orthonormal basis $\left\{e_{i}, i \in I\right\}$ in $\mathcal{H}$. Then the family $\left\{\epsilon_{i}, i \in I\right\}$, defined by $T_{\phi} \epsilon_{i}=e_{i}, \forall \in I$, forms an orthonormal family in $K_{\phi}(X, \mu)$, since one has $\left\langle\epsilon_{i} \mid \epsilon_{k}\right\rangle_{\phi}=\left\langle T_{\phi} \epsilon_{i} \mid T_{\phi} \epsilon_{k}\right\rangle=\left\langle e_{i} \mid e_{k}\right\rangle=\delta_{i k}$. Thus $\left\{\epsilon_{i}, i \in I\right\}$ forms an orthonormal basis in

$$
\mathcal{H}_{K}^{\phi}:=\overline{\operatorname{span}\left\{\epsilon_{i}: i \in I\right\}}\|\cdot\|_{\phi} .
$$

By [22] (Theorem 23), it follows that $\mathcal{H}_{K}^{\phi}$ is a reproducing kernel Hilbert space and one has (compare (20))

$$
\mathcal{H}_{K}^{\phi} \simeq W_{\phi}(X, \mu) \simeq\left(\operatorname{Ran} C_{\psi},\|\cdot\|_{\phi}\right)
$$

Finally, the reproducing kernel of $\mathcal{H}_{K}^{\phi}$ is given by

$$
\mathcal{K}_{\phi}(x, y)=\left\langle S_{\phi, \psi}^{-1} \psi_{x} \mid S_{\phi, \psi}^{-1} \psi_{y}\right\rangle
$$

Actually, the kernel $\mathcal{R}_{\psi, \phi}(x, y):=\left\langle S_{\psi, \phi}^{-1} \phi_{y} \mid \psi_{x}\right\rangle$ is also reproducing, but for the inner product of $L^{2}(X, \mu)$, whereas (25) is reproducing for the inner product $\langle\cdot \mid \cdot\rangle_{\phi}$ and is therefore the correct reproducing kernel of $\mathcal{H}_{K}^{\phi}$.

\section{The Case $Y=\mathcal{H}$, a Hilbert Space, and $Z=V$, a PIP-Space}

Clearly, the inner product (24) need not always be defined, it is a partial inner product on the space of measurable functions on $(X, \mu)$. This obviously suggests to take for the target space $Z(X, \mu)$ 
a PIP-space $V \sim\left\{V_{p}(X, \mu)\right\}$ around $L^{2}(X, \mu)$, as we have proposed in [23], that we mostly follow in this section. As for the relevant notions about PIP-spaces, we have collected them in the Appendix A.

\subsection{The Case Where Z Is a Rigged Hilbert Space}

The simplest example of a PIP-space is a rigged Hilbert space (RHS). Let indeed $\mathcal{D}[t] \subset \mathcal{H} \subset$ $\mathcal{D}^{\times}\left[t^{\times}\right]$be a RHS with $\mathcal{D}[t]$ reflexive (hence $t$ and $t^{\times}$coincide with the respective Mackey topologies). Given the measure space $(X, \mu)$, we denote by $\langle\cdot, \cdot\rangle$ the sesquilinear form expressing the duality between $\mathcal{D}$ and $\mathcal{D}^{\times}$. This form replaces the inner product $\langle\cdot \mid \cdot\rangle$ used so far. As usual, we suppose that this sesquilinear form extends the inner product of $\mathcal{D}$ (and $\mathcal{H}$ ). This allows to build the triplet above.

Let $x \in X \mapsto \psi_{x}, x \in X \mapsto \phi_{x}$ be weakly measurable functions from $X$ into $\mathcal{D}^{\times}$. Instead of (11), we consider the sesquilinear form

$$
\Omega_{\psi, \phi}^{\mathcal{D}}(f, g)=\int_{X}\left\langle f, \psi_{x}\right\rangle\left\langle\phi_{x}, g\right\rangle \mathrm{d} \mu(x), f, g \in \mathcal{D}
$$

and we assume that it is jointly continuous on $\mathcal{D} \times \mathcal{D}$. Writing

$$
\left\langle S_{\psi, \phi} f, g\right\rangle:=\int_{X}\left\langle f, \psi_{x}\right\rangle\left\langle\phi_{x}, g\right\rangle \mathrm{d} \mu(x), \forall f, g \in \mathcal{D},
$$

we see that the operator $S_{\psi, \phi}$ belongs to $\mathcal{L}\left(\mathcal{D}, \mathcal{D}^{\times}\right)$, the space of all continuous linear maps from $\mathcal{D}$ into $\mathcal{D}^{\times}$.

At this point, we have a choice. A first possibility is to require that the sesquilinear form $\Omega^{\mathcal{D}}$ be well-defined and bounded on $\mathcal{D} \times \mathcal{D}$ in the topology of $\mathcal{H}$. Then $\Omega_{\psi, \phi}^{\mathcal{D}}$ extends to a bounded sesquilinear form on $\mathcal{H} \times \mathcal{H}$ and the discussion of Section 3 may be essentially repeated verbatim. Thus this choice gives almost nothing new.

Another possibility is to assume that the form $\Omega^{\mathcal{D}}$ is jointly continuous on $\mathcal{D} \times \mathcal{D}$, with no other regularity requirement. In that case, the vector space $K_{\phi}(X, \mu)$ must be defined differently, taking into account the (locally convex) topology of $\mathcal{D}$ and $\mathcal{D}^{\times}$. Instead of (13), we require that the integral $\int_{X} \xi(x)\left\langle\phi_{x}, g\right\rangle \mathrm{d} \mu(x)$ exists for every $g \in \mathcal{D}$ and defines a continuous conjugate linear functional on $\mathcal{D}$. As before, we define (weakly) a linear map $T_{\phi}: K_{\phi}(X, \mu) \rightarrow \mathcal{D}^{\times}$by the following relation

$$
\left\langle T_{\phi} \xi, g\right\rangle=\int_{X} \xi(x)\left\langle\phi_{x}, g\right\rangle \mathrm{d} \mu(x), \forall \xi \in K_{\phi}(X, \mu), g \in \mathcal{D}
$$

Next, we define again the vector space $W_{\phi}(X, \mu)=K_{\phi}(X, \mu) / \operatorname{Ker} T_{\phi}$. In order to introduce a topology on $W_{\phi}(X, \mu)$, we consider a bounded subset $\mathcal{M}$ of $\mathcal{D}[t]$ and we define the seminorm

$$
\widehat{\mathrm{p}}_{\mathcal{M}}\left(\xi_{\phi}\right):=\sup _{g \in \mathcal{M}}\left|\left\langle T_{\phi} \xi, g\right\rangle\right|
$$

This means, we are defining the topology of $W_{\phi}(X, \mu)$ by means of the strong dual topology $t^{\times}$of $\mathcal{D}^{\times}$ which we recall is defined by the seminorms

$$
\|F\|_{\mathcal{M}}=\sup _{g \in \mathcal{M}}|\langle F \mid g\rangle|, \quad F \in \mathcal{D}^{\times}
$$

where $\mathcal{M}$ runs over the family of bounded subsets of $\mathcal{D}[t]$. Thus $W_{\phi}(X, \mu)$ is a locally convex space. As said above, the reflexivity of $\mathcal{D}$ entails that $t^{\times}$is equal to the Mackey topology $\tau\left(\mathcal{D}^{\times}, \mathcal{D}\right)$.

Next we consider the dual $W_{\phi}(X, \mu)^{*}$ of the space $W_{\phi}(X, \mu)$, that is, the set of continuous linear functionals on $W_{\phi}(X, \mu)$. As topology on $W_{\phi}(X, \mu)^{*}$, we take the strong dual topology. This being done, almost all the results of Section 3 may be deduced. In particular, Theorem 1 remains true, under the form. 
Theorem 3. Under the condition (26), every continuous linear functional $F$ on $W_{\phi}(X, \mu)$, i.e., $F \in W_{\phi}(X, \mu)^{*}$, can be represented as

$$
F\left(\xi_{\phi}\right)=\int_{X} \xi(x) \overline{\eta(x)} \mathrm{d} \mu(x), \forall \xi_{\phi} \in W_{\phi}(X, \mu),
$$

with $\eta \in K_{\psi}(X, \mu)$. The residue class $\eta_{\psi} \in W_{\psi}(X, \mu)$ is uniquely determined.

As in the previous case, one may prove again that $W_{\phi}(X, \mu)^{*}$ can be identified with a closed subspace of $\overline{W_{\psi}}(X, \mu):=\left\{\overline{\xi_{\psi}}: \xi \in K_{\psi}(X, \mu)\right\}$, but we cannot go further. Indeed the previous results rely heavily on Hilbert space methods, which are not available here.

\subsection{The Case Where Z Is a LHS/LBS}

The next choice is to take for $Z$ a genuine PIP-space, while keeping for the initial space $Y$ a Hilbert space $\mathcal{H}$. For simplicity, we restrict ourselves to a lattice of Banach spaces (LBS) or a lattice of Hilbert spaces (LHS) [7], which is amply sufficient for applications. For instance, $\left\{L^{p}[0,1], 1<p<\infty\right\}$, the lattice generated by $\left\{L^{p}(\mathbb{R}), 1<p<\infty\right\}$ or a lattice of weighted $L^{2}$ spaces.

Let $V_{J}=\left\{V_{p}(X, \mu), p \in J\right\}$ be a LBS or a LHS of $\mu$-measurable functions with the property

$$
\xi \in V_{p}, \eta \in V_{\bar{p}} \Longrightarrow \xi \bar{\eta} \in L^{1}(X, \mu) \quad \text { and } \quad\left|\int_{X} \xi(x) \overline{\eta(x)} \mathrm{d} \mu(x)\right| \leq\|\xi\|_{p}\|\eta\|_{\bar{p}} .
$$

Thus the central Hilbert space is $V_{o}:=L^{2}(X, \mu)$ and the spaces $V_{p}:=V_{p}(X, \mu), V_{\bar{p}}:=V_{\bar{p}}(X, \mu)$ are dual of each other with respect to the $L^{2}$ inner product. The partial inner product, which extends the inner product of $L^{2}(X, \mu)$, is denoted again by $\langle\cdot \mid \cdot\rangle$. As usual we put $V=\sum_{p \in J} V_{p}$ and $V^{\#}=\bigcap_{p \in J} V_{p}$. According to the general theory of PIP-spaces [7], the vector space $V$ is the algebraic inductive limit of the $V_{p}$ 's (see the Appendix A.1). Thus $f \in V$ means that $f \in V_{p}$ for some $p \in J$.

Let $\psi, \phi$ be weakly measurable functions from $X$ into $\mathcal{H}$. In the case of Section 3 , if $(\psi, \phi)$ is a reproducing pair, the frame operator may be written as

$$
\left\langle S_{\psi, \phi} f \mid g\right\rangle=\int_{X}\left\langle f \mid \psi_{x}\right\rangle\left\langle\phi_{x} \mid g\right\rangle \mathrm{d} \mu(x)=\int_{X} C_{\psi} f(x) \overline{C_{\phi} g(x)} \mathrm{d} \mu(x),
$$

which is well defined for all $f, g \in \mathcal{H}$. But now the inner product is only partial, and this motivates the next assumption. In view of (31), (32), and the definition of $V$, we assume that the following condition holds:

(p) $\exists p \in J$ such that $C_{\psi} f=\langle f \mid \psi.\rangle \in V_{p}$ and $C_{\phi} g=\langle g \mid \phi.\rangle \in V_{\bar{p}}, \forall f, g \in \mathcal{H}$.

We recall that $V_{\bar{p}}$ is the conjugate dual of $V_{p}$. In this case, then

$$
\Omega_{\psi, \phi}(f, g):=\int_{X}\left\langle f \mid \psi_{x}\right\rangle\left\langle\phi_{x} \mid g\right\rangle \mathrm{d} \mu(x), f, g \in \mathcal{H},
$$

defines a sesquilinear form on $\mathcal{H} \times \mathcal{H}$ and one has

$$
\left|\Omega_{\psi, \phi}(f, g)\right| \leq\left\|C_{\psi} f\right\|_{p}\left\|C_{\phi} g\right\|_{\bar{p}}, \forall f, g \in \mathcal{H} .
$$

If $\Omega_{\psi, \phi}$ is bounded as a form on $\mathcal{H} \times \mathcal{H}$ (this is not automatic), there exists a bounded operator $S_{\psi, \phi}$ in $\mathcal{H}$ such that

$$
\left\langle S_{\psi, \phi} f \mid g\right\rangle=\int_{X}\left\langle f \mid \psi_{x}\right\rangle\left\langle\phi_{x} \mid g\right\rangle \mathrm{d} \mu(x), \forall f, g \in \mathcal{H} .
$$

Then $(\psi, \phi)$ is a reproducing pair if $S_{\psi, \phi} \in G L(\mathcal{H})$.

In the sequel, we will often need the map $C_{\psi}: \mathcal{H} \rightarrow V_{r}$ to be continuous. In general, however, we have to assume it explicitly, unless the space $V_{r}$ satisfies additional conditions. 
If $C_{\psi}: \mathcal{H} \rightarrow V_{r}$ is continuous, then $C_{\psi}^{*}: V_{\bar{r}} \rightarrow \mathcal{H}$ exists and it is continuous too. By definition, if $\xi \in V_{\bar{r}}$

$$
\left\langle C_{\psi} f \mid \xi\right\rangle=\int_{X}\left\langle f \mid \psi_{x}\right\rangle \overline{\xi(x)} \mathrm{d} \mu(x), \forall f \in \mathcal{H}
$$

Thus,

$$
C_{\psi}^{*} \xi=\int_{X} \psi_{x} \xi(x) \mathrm{d} \mu(x)
$$

Of course, what we have said about $C_{\psi}$ holds in the very same way for $C_{\phi}$. Assume now that for some $p \in J, C_{\psi}: \mathcal{H} \rightarrow V_{p}$ and $C_{\phi}: \mathcal{H} \rightarrow V_{\bar{p}}$ continuously. Then, $C_{\phi}^{*}: V_{p} \rightarrow \mathcal{H}$ so that $C_{\phi}^{*} C_{\psi}$ is a well-defined bounded operator in $\mathcal{H}$, given by

$$
C_{\phi}^{*} C_{\psi} f=\int_{X}\left\langle f \mid \psi_{x}\right\rangle \phi_{x} \mathrm{~d} \mu(x)=S_{\psi, \phi} f, \quad \forall f \in \mathcal{H},
$$

the last equality following also from (34). Of course, this does not yet imply that $S_{\psi, \phi} \in G L(\mathcal{H})$, thus we do not know whether $(\psi, \phi)$ is a reproducing pair.

Let us now return to the pre-Hilbert space $K_{\phi}(X, \mu)$. First, the defining relation (13) must be written as

$$
\left|\int_{X} \xi(x) \overline{\left(C_{\phi} g\right)(x)} \mathrm{d} \mu(x)\right| \leq c\|g\|, \forall \xi \in K_{\phi}(X, \mu), g \in \mathcal{H} .
$$

Since $C_{\phi}: \mathcal{H} \rightarrow V_{\bar{p}}$, the integral is well defined for all $\xi \in V_{p}$. This means, the inner product on the 1.h.s. is again the partial inner product of $V$. . Hence, we may rewrite (36) as

$$
\left|\left\langle\xi \mid C_{\phi} g\right\rangle\right| \leq c\|g\|, \forall g \in \mathcal{H}, \xi \in V_{p} .
$$

Next, by (31), one has, for $\xi \in V_{p}, g \in \mathcal{H}$,

$$
\left|\left\langle\xi \mid C_{\phi} g\right\rangle\right| \leq\|\xi\|_{p}\left\|C_{\phi} g\right\|_{\bar{p}} \leq c\|\xi\|_{p}\|g\|
$$

where the last inequality follows from the assumption of continuity of $C_{\phi}$. Hence indeed $\xi \in K_{\phi}(X, \mu)$, so that $V_{p} \subset K_{\phi}(X, \mu)$.

As for the adjoint operator, we have $C_{\phi}^{*}: V_{p} \rightarrow \mathcal{H}$. Then we may write, for $\xi \in V_{p}, g \in \mathcal{H}$, $\left\langle\xi \mid C_{\phi} g\right\rangle=\left\langle T_{\phi} \xi \mid g\right\rangle$, thus $C_{\phi}^{*}$ is the restriction from $K_{\phi}(X, \mu)$ to $V_{p}$ of the operator $T_{\phi}: K_{\phi} \rightarrow \mathcal{H}$ given in (14), which reads now as

$$
\left\langle T_{\phi} \xi \mid g\right\rangle=\int_{X} \xi(x)\left\langle\phi_{x} \mid g\right\rangle \mathrm{d} \mu(x), \forall \xi \in V_{p}, g \in \mathcal{H} .
$$

Thus $C_{\phi}^{*}=T_{\phi}\left\lceil V_{p}\right.$.

Next, the construction proceeds as in Section 3. The space $W_{\phi}(X, \mu)=K_{\phi}(X, \mu) / \operatorname{Ker} T_{\phi}$, with the norm $\left\|\xi_{\phi}\right\|_{\phi}=\left\|T_{\phi} \xi\right\|$, is a pre-Hilbert space. Then Theorem 2 remains true, namely,

Theorem 4. If $(\psi, \phi)$ is a reproducing pair, the spaces $W_{\phi}(X, \mu)$ and $W_{\psi}(X, \mu)$ are both Hilbert spaces, conjugate dual of each other with respect to the sesquilinear form (24), namely,

$$
\langle\xi \mid \eta\rangle_{\mu}:=\int_{X} \xi(x) \overline{\eta(x)} \mathrm{d} \mu(x),
$$

which coincides with the inner product of $L^{2}(X, \mu)$ whenever the latter makes sense.

More precisely, we may state 
Proposition 2. Let $(\psi, \phi)$ be a reproducing pair. Then, if $C_{\psi}: \mathcal{H} \rightarrow V_{p}$ and $C_{\phi}: \mathcal{H} \rightarrow V_{\bar{p}}$ continuously, one has

$$
V_{\bar{p}} / \operatorname{Ker} T_{\psi}=W_{\psi}(X, \mu) \simeq \overline{W_{\phi}}(X, \mu)^{*} \text { and } V_{p} / \operatorname{Ker} T_{\phi}=W_{\phi}(X, \mu) \simeq \overline{W_{\psi}}(X, \mu)^{*} .
$$

In these relations, the equality sign means an isomorphism of vector spaces, whereas $\simeq$ denotes an isomorphism of Hilbert spaces.

Since $W_{\psi}(X, \mu)$ and $W_{\phi}(X, \mu)$ are both Hilbert spaces, the relations (38) suggest to take for $V_{p}, V_{\bar{p}}$ Hilbert spaces as well, that is, take for $V$ a LHS. The simplest case is then a Hilbert chain, for instance, the scale (A2) $\left\{\mathcal{H}_{k}, k \in \mathbb{Z}\right\}$ built on the powers of a self-adjoint operator $A>I$.

Choose a reproducing pair $(\psi, \phi)$. Without loss of generality, we suppose that $\psi, \phi$ are dual to each other, that is, $S_{\psi, \phi}=I$. We assume that $\psi$ is an upper semi-frame and $\phi$ is a lower semi-frame, dual to each other. It follows that $C_{\psi}(\mathcal{H}) \subset L^{2}(X, \mu)$. By Condition $(\mathrm{p})$, there is an index $k \geq 1$ such that $C_{\psi}: \mathcal{H} \rightarrow \mathcal{H}_{k}$ and $C_{\phi}: \mathcal{H} \rightarrow \mathcal{H}_{\bar{k}}$ continuously, so that we may identify $V_{p} \equiv \mathcal{H}_{k}$ and $V_{\bar{p}} \equiv \mathcal{H}_{\bar{k}}$. Then, according to Proposition 2, we have $W_{\psi}(X, \mu)=\mathcal{H}_{\bar{k}} / \operatorname{Ker} T_{\psi}$ and $W_{\phi}(X, \mu)=\mathcal{H}_{k} / \operatorname{Ker} T_{\phi}$, as vector spaces. Concrete examples will be given in Section 6.

\section{The Case Where $Y$ and $Z$ Are Both PIP-Spaces}

In the previous sections, we have taken for the initial space $Y$ a single abstract Hilbert space $\mathcal{H}$. Now we will go one step further and take instead an abstract LBS or a LHS $Y \sim\left\{Y_{u}, u \in U\right\}$, with $Y_{o}=\mathcal{H}$, while keeping for $Z$ the space $V_{J} \sim\left\{V_{p}(X, \mu), p \in J\right\}$, a LBS or a LHS of complex measurable functions over the usual measure space $(X, \mu)$, with $V_{o}(X, \mu)=L^{2}(X, \mu)$.

Let $\psi, \phi$ be two weakly measurable functions on $X$, with values in $Y$. In the present context, weak measurability means that, for any $u, v \in U$ such that $\psi \in Y_{u}$ and $\phi \in Y_{v}$, the complex functions $x \in X \mapsto\left\langle f \mid \psi_{x}\right\rangle$ and $x \in X \mapsto\left\langle g \mid \phi_{x}\right\rangle$ are $\mu$-measurable for every $f \in Y_{\bar{u}}, g \in Y_{\bar{v}}$.

Define two analysis operators $C_{\psi}, C_{\phi} \in \mathrm{Op}(Y)$ as follows :

$$
\left(C_{\psi} f\right)(x)=\left\langle f \mid \psi_{x}\right\rangle, f \in Y_{\bar{u}}, \psi_{x} \in Y_{u}, \quad\left(C_{\phi} g\right)(x)=\left\langle g \mid \phi_{x}\right\rangle, g \in Y_{\bar{v}}, \phi_{x} \in Y_{v},
$$

where the inner product is the partial inner product of $Y$.

Definition 1. Given $(u, v)$ such that $\psi \in Y_{u}$ and $\phi \in Y_{v}$, assume there exists $p \in J$ such that

$$
C_{\psi} f \in V_{p}(X, \mu), \forall f \in Y_{\bar{u}}, \quad \text { and } \quad C_{\phi} g \in V_{\bar{p}}(X, \mu), \forall g \in Y_{\bar{v}},
$$

with $C_{\psi}: Y_{\bar{u}} \rightarrow V_{p}(X, \mu)$ and $C_{\phi}: Y_{\bar{v}} \rightarrow V_{\bar{p}}(X, \mu)$ continuous, so that $C_{\psi}^{*}: V_{\bar{p}}(X, \mu) \rightarrow Y_{u}$ and $C_{\phi}^{*}:$ $V_{p}(X, \mu) \rightarrow Y_{v}$ are continuous also. In that case, we say that $(u, v)$ is an admissible couple for $(\psi, \phi)$.

Given an admissible couple $(u, v)$ for $(\psi, \phi)$, the sesquilinear form

$$
\Omega_{\psi, \phi}(f, g)=\int_{X}\left\langle f \mid \psi_{x}\right\rangle\left\langle\phi_{x} \mid g\right\rangle \mathrm{d} \mu=\int_{X}\left(C_{\psi} f\right)(x) \overline{\left(C_{\phi} g\right)(x)} \mathrm{d} \mu
$$

is well-defined on $Y_{\bar{u}} \times Y_{\bar{v}}$ and we have

$$
\left|\Omega_{\psi, \phi}(f, g)\right| \leq\left\|C_{\psi} f\right\|_{p}\left\|C_{\phi} g\right\|_{\bar{p}} \leq c^{\prime}\|f\|_{\bar{u}}\|g\|_{\bar{v}}, \quad \forall f \in Y_{\bar{u}}, g \in Y_{\bar{v}} .
$$

Then there exists a continuous operator $S_{\psi, \phi}: Y_{\bar{u}} \rightarrow Y_{v}$ such that

$$
\Omega_{\psi, \phi}(f, g)=\left\langle S_{\psi, \phi} f \mid g\right\rangle, \quad \forall f \in Y_{\bar{u}}, g \in Y_{\bar{v}} .
$$


More precisely, in the PIP-space operator language (see the Appendix A), $S_{\psi, \phi}$ has a (necessarily continuous) representative $\left[S_{\psi, \phi}\right]_{v \bar{u}}: Y_{\bar{u}} \rightarrow Y_{v}$. It is easily checked that $S_{\psi, \phi}=C_{\phi}^{*} C_{\psi}$. The operator $S_{\psi, \phi}$ is called the frame operator associated to the pair $(\psi, \phi)$.

The map $S_{\psi, \phi}$ has an adjoint $S_{\psi, \phi}^{*}: Y_{\bar{v}} \rightarrow Y_{u}$ defined by

$$
\left\langle S_{\psi, \phi}^{*} g \mid f\right\rangle=\overline{\left\langle S_{\psi, \phi} f \mid g\right\rangle}, \quad f \in Y_{\bar{u}}, g \in Y_{\bar{v}} .
$$

An easy computation shows that

$$
\left\langle S_{\psi, \phi}^{*} g \mid f\right\rangle=\int_{X}\left\langle g \mid \phi_{x}\right\rangle\left\langle\psi_{x} \mid f\right\rangle \mathrm{d} \mu, \quad f \in Y_{\bar{u}}, g \in Y_{\bar{v}} .
$$

Hence, $S_{\psi, \phi}^{*}=S_{\phi, \psi}$.

\subsection{Construction of Coefficient Spaces}

Fix an admissible couple $(u, v)$ for $(\psi, \phi)$. Denote by $K_{\phi}$ the space of all measurable functions $\xi: X \rightarrow \mathbb{C}$ such that the integral

$$
F_{\phi}(g):=\int_{X} \xi(x)\left\langle\phi_{x} \mid g\right\rangle \mathrm{d} \mu
$$

exists for every $g \in Y_{\bar{v}}$ and defines a continuous conjugate linear functional on $Y_{\bar{v}}$. That is, there exists $c>0$ such that

$$
\left|\int_{X} \xi(x)\left\langle\phi_{x} \mid g\right\rangle \mathrm{d} \mu(x)\right| \leq c\|g\|_{\bar{v}}, \forall g \in Y_{\bar{v}} .
$$

As in the previous cases, we refer to $K_{\phi}$ as the coefficient space of $\phi$. The space $K_{\psi}$ is defined in a similar way.

Since the sesquilinear form $\Omega_{\psi, \phi}$ is bounded, by (40), it is clear that all functions $\xi(x)=\left\langle f \mid \psi_{x}\right\rangle$ belong to $K_{\phi}$ since, by assumption,

$$
\int_{X}\left\langle f \mid \psi_{x}\right\rangle\left\langle\phi_{x} \mid g\right\rangle \mathrm{d} \mu(x)
$$

exists and is bounded.

Then, following the familiar pattern, we can define a linear map $T_{\phi}: K_{\phi} \rightarrow Y_{v}$, which we call again the synthesis operator by the following relation

$$
\left\langle T_{\phi} \xi \mid g\right\rangle=\int_{X} \xi(x)\left\langle\phi_{x} \mid g\right\rangle \mathrm{d} \mu, \quad \xi \in K_{\phi}, g \in Y_{\bar{v}}
$$

Set $W_{\phi}=K_{\phi} / \operatorname{Ker} T_{\phi}$ and $\xi_{\phi}:=\xi+\operatorname{Ker} T_{\phi}$. By definition, $\phi$ is called $\mu$-independent whenever Ker $T_{\phi}=\{0\}$; in that case, of course, $W_{\phi}=K_{\phi}$.

The space $W_{\phi}$ can be made into a normed space by defining

$$
\left\|\xi_{\phi}\right\|_{\phi}=\left\|T_{\phi} \xi\right\|_{v}, \xi \in K_{\phi},
$$

which implies that the operator $\widehat{T}_{\phi}: W_{\phi} \rightarrow Y_{v}$ defined by $\widehat{T}_{\phi} \xi_{\phi}:=T_{\phi} \xi$ is continuous, injective and an isometry.

Proposition 3. The space $W_{\phi}$ is a Banach space if, and only if, Ran $T_{\phi}$ is a closed subspace of $Y_{v}$.

Proof. Suppose that $\operatorname{Ran} T_{\phi}$ is closed and let $\left\{\left(\xi_{n}\right)_{\phi}\right\}$ be a Cauchy sequence in $W_{\phi}\left[\|\cdot\|_{\phi}\right]$. Then, by definition $\left\{T_{\phi} \xi_{n}\right\}$ is a Cauchy sequence in $Y_{v}$; hence it converges to $h \in Y_{v}$. Since Ran $T_{\phi}$ is closed, $h=T_{\phi} \xi$ for some $\xi \in K_{\phi}$. It is clear that if $\xi^{\prime} \in K_{\phi}$ is another function such that $h=T_{\phi} \xi^{\prime}$, then $T_{\phi}\left(\xi-\xi^{\prime}\right)=0$ and then $\xi_{\phi}=\xi_{\phi}^{\prime}$. Moreover

$$
\left\|\left(\xi_{n}\right)_{\phi}-\xi_{\phi}\right\|_{\phi}=\left\|T_{\phi} \xi_{n}-T_{\phi} \xi\right\|_{v}=\left\|T_{\phi} \xi_{n}-h\right\|_{v} \rightarrow 0 .
$$


This proves that $W_{\phi}$ is complete. Conversely, suppose that $W_{\phi}$ is a Banach space and let $h=$ $\lim _{n \rightarrow \infty} T_{\phi} \xi_{n}, \xi_{n} \in K_{\phi}$. Then

$$
\left\|\left(\xi_{n}\right)_{\phi}-\left(\xi_{m}\right)_{\phi}\right\|_{\phi}=\left\|T_{\phi} \xi_{n}-T_{\phi} \xi_{m}\right\|_{v} \rightarrow 0 .
$$

Hence there exists $\xi \in K_{\phi}$ such that $\left\|\left(\xi_{n}\right)_{\phi}-\xi_{\phi}\right\| \rightarrow 0$. This in turn implies that $\left\|T_{\phi} \xi_{n}-T_{\phi} \xi\right\| \rightarrow 0$ and, therefore $h=T_{\phi} \xi$. We conclude that $\operatorname{Ran} T_{\phi}$ is closed.

Thus, if $W_{\phi}$ is complete, it has a closed image and its inverse is also continuous (but not necessarily everywhere defined).

Note that, if $Y$ is a LHS, the norm $\|\cdot\|_{\phi}$ is Hilbertian, so that $K_{\phi}\left[\|\cdot\|_{\phi}\right]$ is a pre-Hilbert space (same argument as in Section 3).

The same result applies to $W_{\psi}$, which is also a pre-Hilbert space in the LHS case, i.e., $\widehat{T}_{\psi}: W_{\psi} \rightarrow Y_{u}$ is an isometry.

It is shown in [23] (Theorem 3.4), that a linear functional $F$ on $W_{\phi}\left[\|\cdot\|_{\phi}\right]$ is continuous if, and only if, there exists $g \in Y_{\bar{v}}$ such that

$$
F\left(\xi_{\phi}\right)=\int_{X} \xi(x)\left\langle\phi_{x} \mid g\right\rangle \mathrm{d} \mu, \quad \forall \xi \in K_{\phi},
$$

Then, taking into account the relations (39) and (40) and the fact that $\left\langle\phi_{x} \mid g\right\rangle \in K_{\psi}$, we see that every $F \in W_{\phi}(X, \mu)^{*}$ can be represented as

$$
F\left(\xi_{\phi}\right)=\int_{X} \xi(x) \overline{\eta(x)} \mathrm{d} \mu(x), \forall \xi_{\phi} \in W_{\phi}(X, \mu),
$$

with $\eta \in K_{\psi}(X, \mu)$. Hence, the dual space $W_{\phi}^{*}$ of $W_{\phi}$, with respect to the sesquilinear form given by the $L^{2}$ inner product, can be identified with a space $E_{\phi}$ of measurable functions containing all functions $\left\{\left\langle g \mid \phi_{x}\right\rangle, g \in Y_{\bar{v}}\right\}$.

\subsection{Compatible Pairs}

Fix an admissible couple $(u, v)$ for $(\psi, \phi)$. As seen above, the sesquilinear form

$$
\Omega_{\psi, \phi}(f, g)=\int_{X}\left\langle f \mid \psi_{x}\right\rangle\left\langle\phi_{x} \mid g\right\rangle \mathrm{d} \mu=\int_{X}\left(C_{\psi} f\right)(x) \overline{\left(C_{\phi} g\right)(x)} \mathrm{d} \mu
$$

is well defined and bounded on $Y_{\bar{u}} \times Y_{\bar{v}}$. With a proof similar to that of [23] (Theorem 3.6) or that of Section 4 , one shows that the dual $W_{\phi}^{*}$ can be identified with a closed subspace of $\overline{W_{\psi}}$, the space of conjugates of elements of $W_{\psi}$. On the other hand, it was proved in [26] that, for a reproducing pair of $\mathcal{H}$-valued weakly measurable functions $(\psi, \phi)$, the spaces $W_{\phi}$ and $W_{\psi}$ are both Hilbert spaces in conjugate duality to each other (see Theorem 2).

In the same vein as in Section 3, in particular Theorem 2, we define a new concept, generalizing to the present situation what has been done in [25].

Definition 2. Let $(u, v)$ be an admissible couple for $(\psi, \phi)$. We say that $\psi, \phi$ are compatible if $W_{\phi}^{*}$ is topologically isomorphic to $\overline{W_{\psi}}$ and $W_{\psi}^{\times}$, the conjugate dual of $W_{\psi}$, is topologically isomorphic to $W_{\phi}$. Thus we identify the dual $W_{\phi}^{*}$ with $\overline{W_{\psi}}$ and the conjugate dual $W_{\psi}^{\times}$with $W_{\phi}$.

This definition implies that the spaces $W_{\phi}$ and $W_{\psi}$ are reflexive spaces enjoying the duality properties mentioned above, which we write shortly as $W_{\phi}^{*} \approx \bar{W}_{\psi}$ and $W_{\psi}^{\times} \approx W_{\phi}$. Then we have

$$
W_{\psi}^{*} \approx \overline{W_{\psi}^{\times}} \approx \overline{W_{\phi}} \text { and } W_{\phi}^{\times} \approx \overline{W_{\psi}^{*}} \approx W_{\psi} .
$$

This proves that $(\phi, \psi)$ is a compatible pair if and only if $(\psi, \phi)$ is a compatible pair. 
In what follows, for simplicity, we will consider pairs $(\psi, \phi)$ with $\psi, \phi$ both $\mu$-independent. In this case $W_{\psi}=K_{\psi}, W_{\phi}=K_{\phi}$. Hence we will use the notation $K_{\psi}, K_{\phi}$ for these spaces.

As a matter of fact, compatible pairs and reproducing pairs are closely related, as shown in the following result.

Theorem 5. Let $(u, v)$ be an admissible couple for $(\psi, \phi)$, with $\psi, \phi$ both $\mu$-independent and $\mu$-total. Consider the following statements :

(i) $(\psi, \phi)$ is a compatible pair;

(ii) The operator $S_{\psi, \phi}: Y_{\bar{u}} \rightarrow Y_{v}$ is bounded with bounded inverse, that is, $(\psi, \phi)$ is a reproducing pair.

Then, if $K_{\phi}$ is complete, (i) implies (ii). On the other hand, (ii) always implies (i). Thus, if $K_{\phi}$ is complete, the two statements are equivalent.

Proof. Since $\psi, \phi$ are $\mu$-independent, $\widehat{T}_{\phi}=T_{\phi}$.

(i) $\Rightarrow$ (ii): If $(u, v)$ is an admissible couple for $(\psi, \phi)$ the operator $S_{\psi, \phi}$ is continuous from $Y_{\bar{u}}$ into $Y_{v}$. It is injective, since $\phi$ is $\mu$-independent and $\psi$ is $\mu$-total. Next we show that it is also surjective. We first prove that $\operatorname{Ran} T_{\phi}$ is dense in $Y_{v}$. Suppose that $g \in Y_{\bar{v}}$ is such that $\left\langle T_{\phi} \xi \mid g\right\rangle=0$ for every $\xi \in K_{\phi}$. Thus,

$$
\left\langle T_{\phi} \xi \mid g\right\rangle=\int_{X} \xi(x)\left\langle\phi_{x} \mid g\right\rangle \mathrm{d} \mu=0, \quad \forall \xi \in K_{\phi} .
$$

In particular this is true if we take $\xi(x)=\left\langle f \mid \psi_{x}\right\rangle$, with $f \in Y_{\bar{u}}$. The $\mu$-independence of $\psi$ implies that $\left\langle\phi_{x} \mid g\right\rangle=0$ for almost every $x \in X$. Hence $g=0$, because $\phi$ is $\mu$-total.

By Proposition 3, Ran $T_{\phi}$ is closed in $Y_{v}$. Hence, Ran $T_{\phi}=Y_{v}$. Therefore, for every $\Phi \in Y_{v}$ there exists a unique $\xi \in K_{\phi}$ such that $T_{\phi} \xi=\Phi$. This implies that:

$$
\langle\Phi \mid g\rangle=\int_{X} \xi(x)\left\langle\phi_{x} \mid g\right\rangle \mathrm{d} \mu, \quad \forall g \in Y_{\bar{v}} .
$$

By the definition of compatible pairs, the conjugate linear functional $F_{\xi}$ on $K_{\psi}$ defined by:

$$
F_{\xi}(\eta)=\int_{X} \xi(x) \overline{\eta(x)} \mathrm{d} \mu, \quad \eta \in K_{\psi} .
$$

is continuous. Then, as discussed at the end of Section 5.1, there exists a unique $f \in Y_{\bar{u}}$ such that

$$
F_{\xi}(\eta)=\int_{X}\left\langle f \mid \psi_{x}\right\rangle \overline{\eta(x)} \mathrm{d} \mu, \quad \eta \in K_{\psi} .
$$

Hence, in particular

$$
F_{\xi}\left(\left\langle g \mid \phi_{x}\right\rangle\right)=\int_{X}\left\langle f \mid \psi_{x}\right\rangle\left\langle\phi_{x} \mid g\right\rangle \mathrm{d} \mu, \quad \forall g \in Y_{\bar{v}}
$$

In conclusion,

$$
\langle\Phi \mid g\rangle=\int_{X}\left\langle f \mid \psi_{x}\right\rangle\left\langle\phi_{x} \mid g\right\rangle \mathrm{d} \mu, \quad \forall g \in Y_{\bar{v}} .
$$

This implies that $\Phi=S_{\psi, \phi} f$. Hence $S_{\psi, \phi}^{-1}$ is bounded, by the inverse mapping theorem.

(ii) $\Rightarrow$ (i): Let $H \in K_{\phi}^{*}$, the dual of $K_{\phi}$; then, as discussed at the end of Section 5.1 there exists $g \in Y_{\bar{v}}$ such that:

$$
H(\xi)=H_{g}(\xi)=\int_{X} \xi(x)\left\langle\phi_{x} \mid g\right\rangle \mathrm{d} \mu, \quad \forall \xi \in K_{\phi} .
$$

We show that $g$ is unique. Suppose that there exists another $g^{\prime}$ satisfying the same condition. Then it follows that:

$$
\int_{X} \xi(x)\left\langle\phi_{x} \mid g-g^{\prime}\right\rangle \mathrm{d} \mu=0, \quad \forall \xi \in K_{\phi} .
$$


In particular, this is true for $\xi(x)=\left\langle f \mid \psi_{x}\right\rangle$, for every $f \in Y_{\bar{u}}$. Thus:

$$
\int_{X}\left\langle f \mid \psi_{x}\right\rangle\left\langle\phi_{x} \mid g-g^{\prime}\right\rangle \mathrm{d} \mu=0, \quad \forall \xi \in K_{\phi} .
$$

Hence, $\left\langle S_{\psi, \phi} f \mid g-g^{\prime}\right\rangle=0$, for every $f \in Y_{\bar{u}}$. However, since $S_{\psi, \phi} Y_{\bar{u}}=Y_{v}$, we conclude that $\left\langle G \mid g-g^{\prime}\right\rangle=0$, for every $G \in Y_{v}$ and this, in turn, implies that $g=g^{\prime}$. Therefore, we can define a map $\Xi: H \in K_{\phi}^{*} \mapsto g \in Y_{\bar{v}}$, where $g$ is the unique element of $Y_{\bar{v}}$ such that $H=H_{g}$. This map is an isomorphism of vector spaces. Indeed, it is clearly injective. On the other hand, if $g \in Y_{\bar{v}}$, the functional

$$
H(\xi)=H_{g}(\xi)=\int_{X} \xi(x)\left\langle\phi_{x} \mid g\right\rangle \mathrm{d} \mu, \quad \xi \in K_{\phi}
$$

is in $K_{\phi}^{*}$ and satisfies $\Xi\left(H_{g}\right)=g$. It is clear that the function $\eta(x)=\left\langle g \mid \phi_{x}\right\rangle$ is an element of $K_{\psi}$. Assume that there exists another function $\eta^{\prime} \in K_{\psi}$ such that

$$
H(\xi)=\int_{X} \xi(x) \overline{\eta^{\prime}(x)} \mathrm{d} \mu, \quad \xi \in K_{\phi}
$$

This implies that:

$$
\int_{X} \xi(x) \overline{\left(\eta(x)-\eta^{\prime}(x)\right)} \mathrm{d} \mu=0, \quad \forall \xi \in K_{\phi} .
$$

Take $\xi(x)=\left\langle f \mid \psi_{x}\right\rangle, f \in Y_{\bar{u}}$, then $\eta-\eta^{\prime} \in \operatorname{Ker} T_{\psi}$. Hence $\eta=\eta^{\prime}$. Then we can define a linear map:

$$
\Theta: H \in K_{\phi}^{*} \mapsto \eta \in K_{\psi},
$$

where $K_{\phi}^{*}$ denote the dual space of $K_{\phi}$. The map $\Theta$ is clearly injective. As we have seen $T_{\phi}^{\times} g=\phi_{g} \in K_{\psi}$, for every $g \in Y_{\bar{v}}$, where $\phi_{g}(x)=\left\langle g \mid \phi_{x}\right\rangle$. Indeed, $T_{\phi}: K_{\phi} \rightarrow Y_{v}$. Hence $T_{\phi}^{*}: Y_{\bar{v}} \rightarrow K_{\phi}^{*}$, thus $T_{\phi}^{\times}: Y_{\bar{v}} \rightarrow K_{\phi}^{\times} \approx K_{\psi}$. Now we put $C_{\phi, \psi} g=T_{\phi}^{\times} g$. Hence $C_{\phi, \psi}$ is a conjugate linear map of $Y_{\bar{v}}$ into $K_{\psi}$. We want to prove that $\operatorname{Ran} C_{\phi, \psi}$ coincides with $K_{\psi}$.

First we have

$$
\begin{aligned}
\|g\|_{\bar{v}} & =\left\|S_{\phi, \psi}^{-1} S_{\phi, \psi} g\right\|_{\bar{v}} \leq c\left\|S_{\phi, \psi} g\right\|_{u}=c \sup _{\|h\|_{\bar{u}} \leq 1}\left|\left\langle S_{\phi, \psi} g \mid h\right\rangle\right| \\
& =c \sup _{\|h\|_{\bar{u}} \leq 1}\left|\int_{X}\left\langle g \mid \phi_{x}\right\rangle\left\langle\psi_{x} \mid h\right\rangle \mathrm{d} \mu\right|=c\left\|T_{\psi} \phi_{g}\right\|_{u}=c\left\|\phi_{g}\right\|_{\psi},
\end{aligned}
$$

This inequality implies that $\operatorname{Ran} C_{\phi, \psi}$ is closed in $K_{\psi}$.

Next, we prove that $\operatorname{Ran} C_{\phi, \psi}$ is also dense in $K_{\psi}$. If it was not so, there would be a nonzero continuous linear functional $F$ on $K_{\psi}$ such that $F\left(\left\langle f \mid \phi_{x}\right\rangle\right)=0$ for every $f \in \mathcal{D}$. Hence, there would exist $g \neq 0$ such that:

$$
F(\xi)=\int_{X} \xi(x)\left\langle\psi_{x} \mid g\right\rangle \mathrm{d} \mu, \quad \forall \xi \in K_{\psi}
$$

and therefore

$$
F\left(\phi_{f}\right)=\int_{X}\left\langle f \mid \phi_{x}\right\rangle\left\langle\psi_{x} \mid g\right\rangle \mathrm{d} \mu, \quad \forall f \in Y_{\bar{v}} .
$$

This implies that $\left\langle S_{\phi, \psi} f \mid g\right\rangle=0$ for all $f \in Y_{\bar{v}}$. However, since $S_{\phi, \psi} Y_{\bar{v}}=Y_{u}$, this yields a contradiction. Let us now consider the map $\Theta$ defined in (45). An immediate consequence of the equality $\operatorname{Ran} C_{\phi, \psi}=$ $K_{\psi}$ is that $\Theta$ is surjective. This implies that the conjugate dual of $K_{\phi}$ can be identified with $\overline{K_{\psi}}$, the space of conjugates of elements of $K_{\psi}$. 


\subsection{The General Case}

Take again an admissible couple $(u, v)$ for $(\psi, \phi)$, assuming both $\phi, \psi$ to be $\mu$-independent and $\mu$-total, for simplicity. We know from Section 5.2 that the dual $K_{\phi}^{*}$ can be identified with a closed subspace of $\overline{K_{\psi}}$, the space of conjugates of elements of $K_{\phi}$. In addition, $K_{\phi}^{*}$ is complete as a dual.

Assume first that $(\psi, \phi)$ is a compatible pair. Then we have $K_{\phi}^{*}=\overline{K_{\psi}}$ and $K_{\psi}^{*}=\overline{K_{\phi}}$. Notice that both spaces $K_{\psi}$ and $K_{\phi}$ are Banach spaces in general, but they are Hilbert spaces in the case of a LHS since then the norm (43) is Hilbertian [26]. Now $T_{\psi}: K_{\psi} \rightarrow Y_{u}$ and $T_{\phi}: K_{\phi} \rightarrow Y_{v}$ are continuous and bijective, hence the inverse maps $T_{\psi}^{-1}: Y_{u} \rightarrow K_{\psi}$ and $T_{\phi}^{-1}: Y_{v} \rightarrow K_{\phi}$ are also continuous by the inverse mapping theorem. Thus $T_{\psi}$ and $T_{\phi}$ are isomorphisms. In addition, they are isometries, by (43). Thus $K_{\psi}$ et $Y_{u}$ are isometric and so are the respective duals $K_{\psi}^{*}$ and $Y_{\bar{v}}$ and the same for $K_{\phi} \sim Y_{v}$. Since $K_{\phi}^{*}=\overline{K_{\psi}}$ (as spaces of measurable functions), we can say that $Y_{\bar{v}}$ and $\overline{Y_{u}}$ have equal norms, hence we have $\bar{v}=u$. Thus this is a necessary condition for $(\psi, \phi)$ to be a compatible pair for the admissible couple $(u, v)$.

If the condition $\bar{v}=u$ is satisfied, it follows that the frame operator $S_{\psi, \phi}$ maps $Y_{v}$ onto itself continuously and has a bounded inverse, since the representative $\left[S_{\psi, \phi}\right]_{v v}$ is invertible, which means that $S_{\psi, \phi}$ is an invertible PIP-space operator [28] (see Appendix A.2).

More generally, given an admissible couple $(u, v)$ for $(\psi, \phi)$, we may ask under which conditions the latter is a compatible pair, which is the same thing as a reproducing pair, according to Theorem 5 . First, we need $\bar{v}=u$, that is, there must exist $p \in J$ such that $C_{\psi}: Y_{u} \rightarrow V_{p}(X)$ and $C_{\phi}: Y_{\bar{u}} \rightarrow V_{\bar{p}}(X)$ continuously. In that case, since $\left[S_{\psi, \phi}\right]_{v v}$ exists, so are the representatives $\left[S_{\psi, \phi}\right]_{v^{\prime \prime} v^{\prime}}: Y_{v^{\prime}} \rightarrow Y_{v^{\prime \prime}}$, where $v^{\prime} \leq v, v^{\prime \prime} \geq v$. The required condition is then that one of these representatives $\left[S_{\psi, \phi}\right]_{v^{\prime \prime} v^{\prime}}$ be invertible. This replaces the condition $S_{\psi, \phi} \in G L(\mathcal{H})$ used for reproducing pairs [23,26].

\subsection{Comparison with the Case $Y=\mathcal{H}$}

As we said in Section 3, the case treated in [23] (Section 4) amounts to take for the initial space $Y$ a single Hilbert space $\mathcal{H}$. We will adapt here the results of that paper to the new situation, described in Definition 1.

Let us suppose that the spaces $V_{r}$ have the following property:

(k) If $\xi_{n} \rightarrow \xi$ in $V_{r}$, then, for every compact subset $K \subset X$, there exists a subsequence $\left\{\xi_{n}^{K}\right\}$ of $\left\{\xi_{n}\right\}$ which converges to $\xi$ almost everywhere in $K$.

The property ( $k$ ) is satisfied by $L^{p}$-spaces [29] (Theorem 3.12), but presumably not in general. It is satisfied, however, if one has a continuous embedding of a given Banach space of measurable functions into $L_{\text {loc' }}^{1}$, which applies, for example, to all the Banach function spaces in the sense of Luxemburg-Zaanen [30]. Notice that Condition (k) concerns only the target space $Z$, it is independent of the initial space $Y$.

Let $(u, v)$ be an admissible couple for $(\psi, \phi)$. Instead of $C_{\psi}: \mathcal{H} \rightarrow V$, we have that $\psi_{x} \in Y_{u}, \forall x \in$ $X$, and $C_{\psi}: Y_{\bar{u}} \rightarrow V_{p}(X)$. Let $V_{r}$ be an arbitrary element of $V_{J}$ (an assaying subspace [7], which is a Banach space or a Hilbert space). Following [23] (Section 4), we define

$$
D_{r, \bar{u}}\left(C_{\psi}\right)=\left\{f \in Y_{\bar{u}}: C_{\psi} f \in V_{r}\right\}, r \in J .
$$

In particular, $D_{r, \bar{u}}\left(C_{\psi}\right)=Y_{\bar{u}}$ means $C_{\psi}\left(Y_{\bar{u}}\right) \subset V_{r}$. According to Definition 1, this condition is satisfied for $r=p$, but not necessarily otherwise.

Then we have the following results, corresponding to Proposition 4.2 and Corollaries 4.3 and 4.4 of [23]. 
Proposition 4. Assume that (k) holds for $V_{r}$. Then :

(i) $C_{\psi}: D_{r, \bar{u}}\left(C_{\psi}\right) \rightarrow V_{r}$ is a closed linear map.

(ii) If for some $r \in J, C_{\psi}\left(Y_{\bar{u}}\right) \subset V_{r}$, then $C_{\psi}: Y_{\bar{u}} \rightarrow V_{r}$ is continuous.

(iii) If $C_{\psi}\left(Y_{\bar{u}}\right) \subset V_{p}$ and $C_{\phi}\left(Y_{\bar{v}}\right) \subset V_{\bar{p}}$, the form $\Omega$ is bounded on $Y_{\bar{u}} \times Y_{\bar{v}}$, that is, $\left|\Omega_{\psi, \phi}(f, g)\right| \leq$ $c\|f\|_{\bar{u}}\|g\|_{\bar{v}}$.

Proof. The proof of (i) follows verbatim that of [23] (Proposition 4.2), simply replacing $\mathcal{H}$ by $\Upsilon_{\bar{u}}$. The same is true for (ii) and (iii) with the two corollaries.

By definition, $C_{\psi}: Y_{\bar{u}} \rightarrow V_{p}$ is continuous. If $r \neq p$ and condition (k) holds, $C_{\psi}\left(Y_{\bar{u}}\right) \subset V_{r}$ implies that $C_{\psi}: Y_{\bar{u}} \rightarrow V_{r}$ is continuous. If $r \neq p$ and we do not know whether the condition holds, we will have to assume explicitly that $C_{\psi}: Y_{\bar{u}} \rightarrow V_{r}$ is continuous.

Suppose now that for some $r \in J$, the maps $C_{\psi}: Y_{\bar{u}} \rightarrow V_{r}$ and $C_{\phi}: Y_{\bar{v}} \rightarrow V_{\bar{r}}$ are continuous (automatic for $r=p$ ). Then, $C_{\phi}^{*}: V_{r} \rightarrow Y_{\bar{u}}$, so that $S_{\psi, \phi}:=C_{\phi}^{*} C_{\psi}$ is a well-defined bounded operator from $Y_{\bar{u}}$ to $Y_{v}$. Of course, this does not yet imply that $\left[S_{\psi, \phi}\right]_{v \bar{u}}$, or any other representative, has a bounded inverse. Hence we do not know whether $(\psi, \phi)$ is a reproducing pair, even if we impose the necessary condition $\bar{v}=u$.

\section{Examples}

In this section, we present concrete examples that illustrate all three types of reproducing pairs described in the previous sections.

\subsection{A Purely Hilbertian Reproducing Pair}

Several discrete examples may be found in [26] (Section 6.1). Here, however, we restrict ourselves to continuous examples, which are more interesting.

If $\phi \in L^{2}(\mathbb{R}, \mathrm{d} x)$, consider the continuous wavelet system $\phi_{b, a}(x)=a^{-1 / 2} \phi\left(a^{-1}(x-b)\right), b \in$ $\mathbb{R}, a>0$. Let $\psi, \phi \in L^{2}(\mathbb{R}, \mathrm{d} x)$ and suppose they satisfy the following cross-admissibility condition:

$$
c_{\psi, \phi}:=\int_{\mathbb{R}}|\widehat{\psi}(\omega) \widehat{\phi}(\omega)| \frac{\mathrm{d} \omega}{|\omega|}<\infty,
$$

As shown in [6] (Theorem 10.1), this condition implies the well-known orthogonality relations of the corresponding wavelet transform. Thus, $(\psi, \phi)$ is a reproducing pair for $L^{2}(\mathbb{R}, d x)$ with $S_{\psi, \phi}=c_{\psi, \phi} I$. For $\psi=\phi$, the cross-admissibility condition (46) reduces to the classical admissibility condition

$$
c_{\phi}:=\int_{\mathbb{R}}|\widehat{\phi}(\omega)|^{2} \frac{\mathrm{d} \omega}{|\omega|}<\infty .
$$

Considering the obvious inequalities

$$
\left|c_{\psi, \phi}\right| \leq \int_{\mathbb{R}}|\widehat{\psi}(\omega) \widehat{\phi}(\omega)| \frac{\mathrm{d} \omega}{|\omega|} \leq c_{\phi}^{1 / 2} c_{\psi}^{1 / 2},
$$

we see that condition (46) is automatically satisfied whenever $\phi$ and $\psi$ are both admissible, so that indeed $(\psi, \phi)$ is a reproducing pair.

However, nonadmissible wavelets may also generate reproducing pairs, as shown by the following trivial example. Take the Gaussian window $\phi(x)=e^{-\pi x^{2}}$, then $c_{\phi}=\infty$ which implies that $\phi$ is not a continuous wavelet frame. However, if one defines $\psi \in L^{2}(\mathbb{R}, d x)$ in the Fourier domain via $\widehat{\psi}(\omega)=|\omega| \widehat{\phi}(\omega)$, it follows that $0<c_{\psi, \phi}=\|\phi\|_{2}^{2}<\infty$. Thus $(\psi, \phi)$ is a reproducing pair. This example clearly shows the increasing flexibility obtained when replacing continuous frames by reproducing pairs. 


\subsection{A Reproducing Pair with a PIP-Space Target}

As discussed after Proposition 2, we take for $V$ a LHS, more precisely the scale (A2) built on the powers of the self-adjoint operator $A>1$. Thus, according to the discussion at the end of Section 4.2, there is an index $k \geq 1$ such that $C_{\psi}: \mathcal{H} \rightarrow \mathcal{H}_{k}$ and $C_{\phi}: \mathcal{H} \rightarrow \mathcal{H}_{\bar{k}}$ continuously, where we have

$$
\mathcal{H}_{k} \subset \mathcal{H}_{o}=L^{2}(X, \mu) \subset \mathcal{H}_{\bar{k}}
$$

Actually one may give an explicit example, using a Sobolev-type scale [23] (Section 5). Let $\mathcal{H}_{K}$ be a reproducing kernel Hilbert space (RKHS) of (nice) functions on the measure space $\left(X, \mu_{K}\right)$, with kernel function $k_{x}, x \in X$, that is, $f(x)=\left\langle f \mid k_{x}\right\rangle_{K}, \forall f \in \mathcal{H}_{K}$ (we could also take another measure space $\left(Z, \mu_{K}\right)$ ) [27]. The corresponding reproducing kernel is $\mathcal{K}(x, y)=k_{y}(x)=\left\langle k_{y} \mid k_{x}\right\rangle_{K}$. Choose a weight function $m(x)>1$, the analog of the weight $\left(1+|x|^{2}\right)$ considered in the Sobolev case (see Appendix A.1). Define the Hilbert scale $\mathcal{H}_{r}, r \in \mathbb{Z}$, determined by the multiplication operator $A f(x)=m(x) f(x), \forall x \in X$. Then, for some $n \geq 1$, define the measurable functions $\psi_{x}=k_{x} m^{-n}(x), \phi_{x}=k_{x} m^{n}(x)$, so that $C_{\psi}: \mathcal{H}_{K} \rightarrow \mathcal{H}_{n}, C_{\phi}: \mathcal{H}_{K} \rightarrow \mathcal{H}_{\bar{n}}$ continuously, and $\psi, \phi$ are dual of each other. One has indeed $\left\langle\phi_{x} \mid g\right\rangle_{K}=\left\langle k_{x} m^{n}(x) \mid g\right\rangle_{K}=\left\langle k_{x} \mid g m^{n}(x)\right\rangle_{K}=\overline{g(x)} m^{n}(x) \in \mathcal{H}_{\bar{n}}$ and $\left\langle\psi_{x} \mid g\right\rangle_{K}=\overline{g(x)} m^{-n}(x) \in \mathcal{H}_{n}$, which implies duality. Thus $(\psi, \phi)$ is a reproducing pair with $S_{\psi, \phi}=I$, where $\psi$ is an upper semi-frame and $\phi$ a lower semi-frame.

In this case, one can compute the operators $T_{\psi}, T_{\phi}$ explicitly. For all $\xi \in \mathcal{H}_{n}, g \in \mathcal{H}_{K}$, the definition (37) reads as

$$
\left\langle T_{\phi} \xi \mid g\right\rangle_{K}=\int_{X} \xi(x)\left\langle\phi_{x} \mid g\right\rangle_{K} \mathrm{~d} \mu(x),=\int_{X} \xi(x) \overline{g(x)} m^{n}(x) \mathrm{d} \mu,
$$

that is, $\left(T_{\phi} \xi\right)(x)=\xi(x) m^{n}(x)$ or $T_{\phi} \xi=\xi m^{n}$. However, since the weight $m(x)>1$ is invertible, $\bar{g} m^{n}$ runs over the whole of $\mathcal{H}_{\bar{n}}$ whenever $g$ runs over $H_{K}$. Hence $\xi \in \operatorname{Ker} T_{\phi} \subset \mathcal{H}_{n}$ means that $\left\langle T_{\phi} \xi \mid g\right\rangle_{K}=0, \forall g \in H_{K}$, which implies $\xi=0$, since the duality between $\mathcal{H}_{n}$ and $\mathcal{H}_{\bar{n}}$ is separating. The same reasoning yields $\operatorname{Ker} T_{\psi}=\{0\}$. Therefore $W_{\phi}(X, \mu)=K_{\phi}(X, \mu)=\mathcal{H}_{n}$ and $W_{\psi}(X, \mu)=$ $K_{\psi}(X, \mu)=\mathcal{H}_{\bar{n}}$.

Similar examples concerning spaces of sequences may be found in [23] (Section 5.2), possibly more general, in the sense that the space $V_{J}$ is no longer a Hilbert scale, but a genuine LHS.

\subsection{A Reproducing Pair with Two PIP-Spaces}

An easy example may be derived from the previous one. For the target space $V_{J}$, we keep the same Hilbert scale (A2) built on the powers of the self-adjoint operator $A>1$. For the initial space $Y$, we take a similar Hilbert scale (A2), but around $\mathcal{H}_{K}$, instead of $L^{2}(X, \mu)$. Thus for each $l \geq 1$, we have

$$
\mathcal{H}_{l} \subset \mathcal{H}_{0} \equiv \mathcal{H}_{K} \subset \mathcal{H}_{\bar{l}}
$$

Hence, $f \in \mathcal{H}_{l}$ means that $\int_{X}|f(x)|^{2} m^{2 l}(x) \mathrm{d} \mu_{K}(x)<\infty$.

Given two positive integers $l, n$, we take for analyzing functions $\psi_{x}=k_{x} m^{-(l+n)}(x), \phi_{x}=$ $k_{x} m^{(l+n)}(x)$, where $k_{x}$ is again the reproducing function of $\mathcal{H}_{K}$. Then we have:

- $\quad \psi_{x} \in \mathcal{H}_{l}$ and $\phi_{x} \in \mathcal{H}_{\bar{l}}$; for instance,

$$
\begin{aligned}
\left\|\psi_{x}\right\|_{l}^{2} & =\int_{X}\left|\psi_{x}\right|^{2} m^{2 l}(x) \mathrm{d} \mu_{K}(x)=\int_{X}\left|k_{x}\right|^{2} m^{-2 n}(x) \mathrm{d} \mu_{K}(x) \\
& <\int_{X}\left|k_{x}\right|^{2} \mathrm{~d} \mu_{K}(x)<\infty, \text { since } m(x)>1 .
\end{aligned}
$$

- $\forall f \in \mathcal{H}_{\bar{l}}, C_{\psi} f=\left\langle f \mid \psi_{x}\right\rangle_{K}=f m^{-(l+n)} \in \mathcal{H}_{n}$.

- $\forall g \in \mathcal{H}_{l}, C_{\phi} g=\left\langle g \mid \phi_{x}\right\rangle_{K}=g m^{(l+n)} \in \mathcal{H}_{\bar{n}}$. 
In conclusion, as for the previous example, $(\psi, \phi)$ is a reproducing pair with $S_{\psi, \phi}=I$, where $\psi$ is an upper semi-frame and $\phi$ a lower semi-frame.

Here too, one can compute the operators $T_{\psi}, T_{\phi}$ explicitly. For all $\xi \in \mathcal{H}_{n}, g \in \mathcal{H}_{l}$, the definition (37) reads as

$$
\left\langle T_{\phi} \xi \mid g\right\rangle_{K}=\int_{X} \xi(x)\left\langle\phi_{x} \mid g\right\rangle_{K} \mathrm{~d} \mu_{K}(x)=\int_{X} \xi(x) \overline{g(x)} m^{(l+n)} \mathrm{d} \mu_{K}(x),
$$

that is, $\left(T_{\phi} \xi\right)(x)=\xi(x) m^{(l+n)}(x)$ or $T_{\phi} \xi=\xi m^{(l+n)}$. Similarly $T_{\psi} \xi=\xi m^{-(l+n)}$.

As compared with the general scheme of Definition 1, we have taken $Y_{u} \equiv \mathcal{H}_{l}, Y_{v} \equiv \mathcal{H}_{\bar{l}}, V_{p} \equiv$ $\mathcal{H}_{n}, V_{\bar{p}} \equiv \mathcal{H}_{\bar{n}}$. This means, in particular, that the condition $v=\bar{u}$ is satisfied, as expected for a reproducing pair.

Author Contributions: Both authors, Antoine and Trapani, contributed equally.

Acknowledgments: We thank the referees for their remarks and suggestions, that have significantly clarified and improved the article.

Conflicts of Interest: The authors declare no conflict of interest.

\section{Appendix A. Lattices of Banach or Hilbert Spaces and Operators on Them}

\section{Appendix A.1. Lattices of Banach or Hilbert Spaces}

For the convenience of the reader, we summarize in this Appendix the basic facts concerning PIP-spaces and operators on them. However, we will restrict the discussion to the simpler case of a lattice of Banach spaces (LBS) or Hilbert spaces (LHS). Further information may be found in our monograph [7] or the review paper [18].

Let thus $\mathcal{I}=\left\{V_{p}, p \in I\right\}$ be a family of Hilbert spaces or reflexive Banach spaces, partially ordered by inclusion. Then $\mathcal{I}$ generates an involutive lattice $\mathcal{J}$, indexed by $J$, through the operations $(p, q, r \in I)$ :

$$
\begin{array}{lrll}
\text {. involution: } & V_{r} & \leftrightarrow V_{\bar{r}}=V_{r}^{\times} \text {, the conjugate dual of } V_{r} \\
\text {. infimum: } & V_{p \wedge q}:=V_{p} \wedge V_{q}=V_{p} \cap V_{q} \\
\text {. supremum: } & V_{p \vee q} & :=V_{p} \vee V_{q}=V_{p}+V_{q} .
\end{array}
$$

It turns out that both $V_{p \wedge q}$ and $V_{p \vee q}$ are Hilbert spaces, resp. reflexive Banach spaces, under appropriate norms (the so-called projective, resp. inductive norms). Clearly the index set $J$ is also an involutive lattice, isomorphic to $\mathcal{J}$ by the correspondence $r \leftrightarrow V_{r}$.

Assume that the following conditions are satisfied:

(i) $\mathcal{I}$ contains a unique self-dual, Hilbert subspace $V_{o}=V_{\bar{o}}$.

(ii) for every $V_{r} \in \mathcal{I}$, the norm $\|\cdot\|_{\bar{r}}$ on $V_{\bar{r}}=V_{r}^{\times}$is the conjugate of the norm $\|\cdot\|_{r}$ on $V_{r}$.

Then the PIP-space $V_{J} \sim\left\{V_{q}, q \in J\right\}$ is called a LBS, resp. a LHS. Notice that $\mathcal{J}=\left\{V_{p}, p \in J\right\}$ denotes a family of spaces, whereas $V_{J} \sim\left\{V_{q}, q \in J\right\}$ denotes the associated PIP-space.

In addition to the family $\mathcal{J}=\left\{V_{r}, r \in J\right\}$, it is convenient to consider the two vector spaces $V$ and $V^{\#}$ defined as

$$
V=\sum_{q \in I} V_{q}, \quad V^{\#}=\bigcap_{q \in I} V_{q} .
$$

These two spaces themselves usually do not belong to $\mathcal{J}$. According to the general theory of PIP-spaces [7], $V$ is the algebraic inductive limit of the $V_{p}$ 's and $V^{\#}$ is the projective limit of the $V_{p}$ 's.

We say that two vectors $f, g \in V$ are compatible (This notion is the basis of the theory of PIP-spaces [7], it should not be confused with that of Definition 2 !) if there exists $r \in J$ such that $f \in$ $V_{r}, g \in V_{\bar{r}}$. Then a partial inner product on $V$ is a Hermitian form $\langle\cdot \mid \cdot\rangle$ defined exactly on compatible pairs of vectors. In particular, the partial inner product $\langle\cdot \mid \cdot\rangle$ coincides with the inner product of $V_{o}$ on the latter. A partial inner product space (PIP-space) is a vector space $V$ equipped with a partial inner product. Clearly LBSs and LHSs are particular cases of PIP-spaces. 
From now on, we will assume that our PIP-space $(V,\langle\cdot \mid \cdot\rangle)$ is nondegenerate, that is, $\langle f \mid g\rangle=0$ for all $f \in V^{\#}$ implies $g=0$. As a consequence, $\left(V^{\#}, V\right)$ and every couple $\left(V_{r}, V_{\bar{r}}\right), r \in J$, are a dual pair in the sense of topological vector spaces [31]. In particular, the original norm topology on $V_{r}$ coincides with its Mackey topology $\tau\left(V_{r}, V_{\bar{r}}\right)$, so that indeed its conjugate dual is $\left(V_{r}\right)^{\times}=V_{\bar{r}}, \forall r \in J$. Then, $r<s$ implies $V_{r} \subset V_{s}$, and the embedding operator $E_{s r}: V_{r} \rightarrow V_{S}$ is continuous and has dense range. In particular, $V^{\#}$ is dense in every $V_{r}$. Throughout we also assume the partial inner product to be positive definite, $\langle f \mid f\rangle>0$ whenever $f \neq 0$.

A standard, albeit trivial, example is that of a Rigged Hilbert space (RHS) $\Phi \subset \mathcal{H} \subset \Phi^{\#}$ (it is trivial because the lattice $\mathcal{I}$ contains only three elements).

Familiar concrete examples of PIP-spaces are sequence spaces, with $V=\omega$, the space of all complex sequences $x=\left(x_{n}\right)$, and spaces of locally integrable functions with $V=L_{\text {loc }}^{1}(\mathbb{R}, \mathrm{d} x)$, the space of Lebesgue measurable functions, integrable over compact subsets.

Among LBSs, the simplest example is that of a chain of reflexive Banach spaces. The prototype is the chain $\mathcal{I}=\left\{L^{p}:=L^{p}([0,1] ; d x), 1<p<\infty\right\}$ of Lebesgue spaces over the interval [0,1].

As for a LHS, the simplest example is the Hilbert scale generated by a self-adjoint operator $A>I$ in a Hilbert space $\mathcal{H}_{0}$. Let $\mathcal{H}_{n}$ be $D\left(A^{n}\right)$, the domain of $A^{n}$, equipped with the graph norm $\|f\|_{n}=\left\|A^{n} f\right\|, f \in D\left(A^{n}\right)$, for $n \in \mathbb{N}$ or $n \in \mathbb{R}^{+}$, and $\mathcal{H}_{\bar{n}}:=\mathcal{H}_{-n}=\mathcal{H}_{n}^{\times}$(conjugate dual):

$$
\mathcal{D}^{\infty}(A):=\bigcap_{n} \mathcal{H}_{n} \subset \ldots \subset \mathcal{H}_{2} \subset \mathcal{H}_{1} \subset \mathcal{H}_{0} \subset \mathcal{H}_{\overline{1}} \subset \mathcal{H}_{\overline{2}} \ldots \subset \mathcal{D} \bar{\infty}(A):=\bigcup_{n} \mathcal{H}_{n} .
$$

Note that here the index $n$ may be integer or real, the link between the two cases being established by the spectral theorem for self-adjoint operators. Here again the inner product of $\mathcal{H}_{0}$ extends to each pair $\mathcal{H}_{n}, \mathcal{H}_{-n}$, but on $\mathcal{D}_{\bar{\infty}}(A)$ it yields only a partial inner product. For standard examples, take $\mathcal{H}_{0}=L^{2}(\mathbb{R}, d x)$ and the following operators:

- $\quad\left(A_{\mathrm{p}} f\right)(x)=\left(1+x^{2}\right)^{1 / 2} f(x)$ yields the Fourier transform of the Sobolev spaces $H^{s}(\mathbb{R}), s \in \mathbb{Z}$.

- $\quad\left(A_{\mathrm{m}} f\right)(x)=\left(1-\frac{d^{2}}{d x^{2}}\right)^{1 / 2} f(x)$ yields the Sobolev spaces $H^{s}(\mathbb{R}), s \in \mathbb{Z}$.

- $\left(A_{\mathrm{osc}} f\right)(x)=\left(1+x^{2}-\frac{d^{2}}{d x^{2}}\right) f(x)$ yields the harmonic oscillator representation of the Schwartz space $\mathcal{S}(\mathbb{R})$ of smooth functions of fast decay and its conjugate dual $\mathcal{S}^{\times}(\mathbb{R})$, the space of of tempered distributions.

\section{Appendix A.2. Operators on LBSs and LHSs}

Let $V_{J}$ be an LHS or an LBS. Then an operator on $V_{J}$ is a map $A$ from a subset $\mathcal{D}(A) \subset V$ into $V$, such that

(i) $\mathcal{D}(A)=\bigcup_{q \in \mathrm{d}(A)} V_{q}$, where $\mathrm{d}(A)$ is a nonempty subset of $J$;

(ii) For every $q \in \mathrm{d}(A)$, there exists $p \in J$ such that the restriction of $A$ to $V_{q}$ is a continuous linear map into $V_{p}$ (we denote this restriction by $A_{p q}$ );

(iii) $A$ has no proper extension satisfying (i) and (ii).

We denote by $\operatorname{Op}\left(V_{J},\right)$ the set of all operators on $V_{J}$. The continuous linear operator $A_{p q}: V_{q} \rightarrow V_{p}$ is called a representative of $A$. The properties of $A$ are conveniently described by the set $\mathrm{j}(A)$ of all pairs $(q, p) \in J \times J$ such that $A$ maps $V_{q}$ continuously into $V_{p}$ Thus the operator $A$ may be identified with the collection of its representatives,

$$
A \simeq\left\{A_{p q}: V_{q} \rightarrow V_{p}:(q, p) \in \mathrm{j}(A)\right\}
$$

It is important to notice that an operator is uniquely determined by any of its representatives, in virtue of Property (iii): there are no extensions for PIP-space operators. 
If $A_{p q}$ is a representative of the operator $A$, then so are $A_{p q^{\prime}}=A_{p q} E_{q q^{\prime}}$, for any $q^{\prime}<q$, where $E_{q q^{\prime}}$ is a representative of the unit operator, and also $A_{p^{\prime} q}=E_{p^{\prime} p} A_{p q}$, for any $p^{\prime}>p$. Operators between an LHS/LBS $Y_{K}$ into another one $V_{J}$ are defined in the same way. They are denoted as $\operatorname{Op}\left(Y_{K}, V_{J}\right)$.

Although an operator may be identified with a separately continous sesquilinear form on $V^{\#} \times V^{\#}$, or a conjugate linear continuous map $V^{\#}$ into $V$, it is more useful to keep also the algebraic operations on operators, namely:

(i) Adjoint: every $A \in \mathrm{Op}\left(V_{J}\right)$ has a unique adjoint $\left.A^{\times} \in \mathrm{Op} V_{J}\right)$, defined by

$$
\left\langle A^{\times} y \mid x\right\rangle=\langle y \mid A x\rangle, \text { for } x \in V_{q}, y \in V_{\bar{p}} \text { and } A_{p q} \text { exists. }
$$

that is, $\left(A^{\times}\right)_{\overline{q p}}=\left(A_{p q}\right)^{*}$, where $\left(A_{p q}\right)^{*}: V_{\bar{p}} \rightarrow V_{\bar{q}}$ is the adjoint map of $A_{p q}$. Furthermore, one has $A^{\times \times}=A$, for every $A \in \mathrm{Op}\left(V_{J}\right)$ : no extension is allowed, by the maximality condition (iii) of the definition.

(ii) Partial multiplication: Let $A, B \in \mathrm{Op}\left(V_{J}\right)$. The product $B A$ is defined if and only if there is a continuous factorization through some $V_{r}$ :

$$
V_{q} \stackrel{A}{\rightarrow} V_{r} \stackrel{B}{\rightarrow} V_{p}, \quad \text { i.e., } \quad(B A)_{p q}=B_{p r} A_{r q} .
$$

Several classes of operators are of particular interest, for instance:

(i) Symmetric operators, defined as those operators satisfying the relation $A^{\times}=A$, since these are the ones that may generate self-adjoint operators in the central Hilbert space [7] (Section 3.3).

(ii) Invertible operators: $A$ is invertible if it has at least one invertible representative; in that case, $A$ has a unique inverse operator $A^{-1} \in \mathrm{Op}\left(V_{J}\right)$ [28].

\section{References}

1. Duffin, R.J.; Schaeffer, A.C. A class of nonharmonic Fourier series. Trans. Am. Math. Soc. 1986, 27, 1271-1283. [CrossRef]

2. Daubechies, I.; Grossmann, A.; Meyer, Y. Painless nonorthogonal expansions. J. Math. Phys. 1986, 27, 1271-1283. [CrossRef]

3. Casazza, P.G. The art of frame theory. Taiwan. J. Math. 2000, 4, 129-202. [CrossRef]

4. Christensen, O. An Introduction to Frames and Riesz Bases; Birkhäuser: Boston, MA, USA, 2003.

5. Daubechies, I. Ten Lectures On Wavelets; CBMS-NSF Regional Conference Series in Applied Mathematics; SIAM: Philadelphia, PA, USA, 1992.

6. Gröchenig, K. Foundations of Time-Frequency Analysis; Birkhäuser: Boston, MA, USA, 2001.

7. Antoine, J.-P.; Trapani, C. Partial Inner Product Spaces: Theory and Applications; Lecture Notes in Mathematics; Springer: Berlin, Germany, 2009; Volume 1986.

8. Ali, S.T.; Antoine, J.-P.; Gazeau, J.-P. Square integrability of group representations on homogeneous spaces I. Reproducing triples and frames. Ann. Inst. H. Poincaré 1991, 55, 829-856.

9. Ali, S.T.; Antoine, J.-P.; Gazeau, J.-P. Continuous frames in Hilbert space. Ann. Phys. 1993, 222, 1-37. [CrossRef]

10. Kaiser, G. A Friendly Guide to Wavelets; Birkhäuser: Boston, MA, USA, 1994.

11. Askari-Hemmat, A.; Dehghan, M.A.; Radjabalipour, M. Generalized frames and their redundancy. Proc. Am. Math. Soc. 2000, 129, 1143-1147. [CrossRef]

12. Fornasier, M.; Rauhut, H. Continuous frames, function spaces, and the discretization problem. J. Fourier Anal. Appl. 2005, 11, 245-287. [CrossRef]

13. Gabardo, J.-P.; Han, D. Frames associated with measurable spaces. Adv. Comput. Math. 2003, 18, 127-147. [CrossRef]

14. Rahimi, A.; Najati, A.; Dehghan, Y.N. Continuous frames in Hilbert spaces. Methods Funct. Anal. Topol. 2006, $12,170-182$. 
15. Antoine, J.-P.; Balazs, P. Frames and semi-frames. J. Phys. A Math. Theor. 2011, 44, 205201; Corrigendum in 2011, 44, 479501. [CrossRef]

16. Antoine, J.-P.; Balazs, P. Frames, semi-frames, and Hilbert scales. Numer. Funct. Anal. Optim. 2012, 33, 736-769. [CrossRef]

17. Speckbacher, M.; Balazs, P. Reproducing pairs and the continuous nonstationary Gabor transform on LCA groups. J. Phys. A Math. Theory 2015, 48, 395201. [CrossRef]

18. Antoine, J.-P.; Trapani, C. Beyond frames: Semi-frames and reproducing pairs. In Mathematical Structures and Applications; In Honor of Prof. M. Norbert Hounkonnou; Diagana, T., Toni, B., Eds.; Springer: New York, NY, USA, 2018; pp. 21-59.

19. Bownik, M. Continuous frames and the Kadison-Singer problem. In Coherent States and Their Applications-A Contemporary Panorama; Springer Proceedings in Physics; Antoine, J.-P., Bagarello, F., Gazeau, J.-P., Eds.; Springer: New York, NY, USA, 2018; Volume 205, pp. 63-88.

20. Hosseini Giv, H.; Radjabalipour, M. On the structure and properties of lower bounded analytic frames. Iran. J. Sci. Technol. 2013, 37A3, 227-230.

21. Jakobsen, M.S.; Lemvig, J. Density and duality theorems for regular Gabor frames. J. Funct. Anal. 2015, 270, 229-263. [CrossRef]

22. Speckbacher, M.; Balazs, P. Frames, their relatives and reproducing kernel Hilbert spaces. arXiv 2017, arXiv:1704.02818.

23. Antoine, J.-P.; Trapani, C. Reproducing pairs of measurable functions and partial inner product spaces. Adv. Oper. Theory 2017, 2, 126-146.

24. Bellomonte, G.; Trapani, C. Riesz-like bases in rigged Hilbert spaces. Z. Anal. Anwend. 2016, 35, $243-265$. [CrossRef]

25. Trapani, C.; Triolo, S.; Tschinke, F. Distribution frames and bases. J. Fourier Anal. Appl. 2019. [CrossRef]

26. Antoine, J.-P.; Speckbacher, M.; Trapani, C. Reproducing pairs of measurable functions. Acta Appl. Math. 2017, 150, 81-101. [CrossRef]

27. Paulsen, V.I.; Raghupathi, M. An Introduction to the Theory of Reproducing Kernel Hilbert Spaces; Cambridge University Press: Cambridge, UK, 2016.

28. Antoine, J.-P.; Trapani, C. Operators on partial inner product spaces: Towards a spectral analysis. Mediterr. J. Math. 2016, 13, 323-351. [CrossRef]

29. Rudin,W. Real and Complex Analysis; McGraw Hill: London, UK; New York, NY, USA, 1987.

30. Luxemburg, W.A.J.; Zaanen, A.C. Some examples of normed Köthe spaces. Math. Ann. 1966, 162, $337-350$. [CrossRef]

31. Köthe, G. Topological Vector Spaces; Springer: Berlin, Germany, 1969; Volume I. 